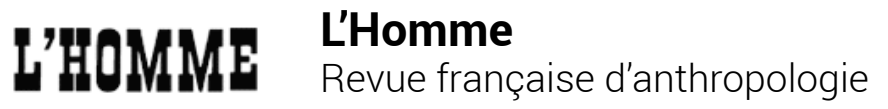 \\ 165 | janvier-mars 2003 \\ Image et anthropologie
}

\section{Warburg, de Kant à Boas}

\section{Claude Imbert}

\section{(Q) OpenEdition \\ Journals}

\section{Édition électronique}

URL : http://journals.openedition.org//homme/197

DOI : 10.4000//homme.197

ISSN : 1953-8103

\section{Éditeur}

Éditions de l'EHESS

\section{Édition imprimée}

Date de publication : 1 janvier 2003

Pagination : 11-40

ISBN : 2-7132-1779-2

ISSN : 0439-4216

\section{Référence électronique}

Claude Imbert, «Warburg, de Kant à Boas », L'Homme [En ligne], 165 | janvier-mars 2003, mis en ligne le 27 mars 2008, consulté le 19 avril 2019. URL : http://journals.openedition.org//homme/197 ; DOI :

10.4000/lhomme.197 


\title{
Warburg, de Kant à Boas
}

\author{
Claude Imbert
}

$D$

E 1927 à 1929, Warburg s'est dédié à son dernier projet, un Atlas d'images, Mnemosyne. En témoignent aujourd'hui un abondant dossier d'archives, une conférence introductive prononcée à la bibliothèque Hertziana en 1929, et cet étonnant montage d'environ soixante-dix écrans de drap noir sur lesquels sont disposées, en des configurations que Warburg remaniera plus d'une fois, de simples photographies de format modeste - environ un millier ${ }^{1}$. Les unes sont des reproductions d'œuvres déjà inventoriées par l'histoire de l'art: peintures, gravures, sculptures, monuments ou dessins provenant de l'Antiquité ou de la Renaissance. Les autres sont des instantanés pris de la vie quotidienne ou de la presse d'information, que ce contexte arrache à leur sens premier de fait divers ou d'événements civils. On y trouve encore quelques photographies de timbresposte ou de publicités. Cet assemblage a surpris, nourrissant plus de doute que d'enthousiasme. Le titre et le sous-titre faisaient doublement paradoxe : une cartographie devait traiter de l'orientation et de la migration plutôt que de la représentation, Mnemosyne évoquait tout autre chose que l'éducation du goût. En un mot : toute une expérience, ce dont Warburg fit son préambule :

« Introduire une distance entre moi et le monde extérieur, c'est cela même que nous pouvons sans aucun doute désigner comme l'acte fondateur de la civilisation humaine. Et si l'espace ainsi ouve rt devient le substrat d'une création artistique, la conscience de la distance peut donner lieu à une durable fonction sociale dont la réussite ou l'échec comme moyen d'orientation intellectuelle sont équivalents au destin de la culture humaine. ${ }^{2}$

1. En attendant l'édition critique annoncée, on peut consulter le coffret publié par les soins de Italo Spinelli \& Roberto Venuti (1998), à l'occasion de l'exposition en Italie, et celui publié à Hambourg par Dölling und Galitz, à l'occasion de l'exposition viennoise (Warburg 1998). Demeurent indispensables E. H. Gombrich (1970) ; l'introduction de Kurt W. Forster à The Renewal of Pagan Antiquity (1999), et les articles et livres de Salvatore Settis (1997, 1999). Également, en langue française, Philippe-Alain Michaud (1998). Nous remercions ici le Warburg Institute de nous avoir autorisés à reproduire les planches et la photo.

2. Formule qui reprend "Pagan-Antique Prophecy in Words and Images in the Age of Luther", dite Conférence Franz Boll (Warburg 1999a), où Warburg considère «l'histoire de l'astronomie et de .../... 
Saxl n'a pas dissimulé sa perplexité quant à cette «tentative fondatrice pour unir une vision philosophique et une vision historique des images ". Ces deux composantes sont, il est vrai, de premièreévidence. Elles caractérisent les travaux de Warburg sur plus de trente années, et Gombrich le confirme amplement. Mais s'agissait-il d'unir encore une fois deux manières de penser l'image qui, nouées dans une Renaissance conçue comme accomplissement de l'Antique, avaient pour longtemps décidé de la nature de l'art? Ou, tout à l'inverse, de les disjoindre pour faire droit à un tertium quid dont l'Atlas proposait un paradigme opaque et laissé inachevé ?

Les quelque soixante dix planches numérotées sont précédées de trois autres, indexées alphabétiquement. C'est également par des lettres que Warburg a distingué les différents points de sa conférence. Ici, les entrées alphabétiques lui donnaient plus de liberté, et surtout une articulation plus explicite que la rhétorique d'un exposé continu, organisé en moments enchaînés. Les planches alphabétiques faisaient voir, comme une axiomatique ou une décomposition vectorielle, les axes en fonction desquels se construit la démonstration. N'y verrait-on qu'une présentation syntagmatique commode et arbitraire, elle n’en aurait pas moins l' a va ntage d’être homogène aux écrans, postulée dans leurs propres termes et dimensions. "Je ne peux plus supporter de voir mes anciennes expressions imprimées sur du papier de luxe" (Joumal, 5/9/1929). Ou t re une insatisfaction dont l' i n ventivité fébrile de Warburg était coutumière, s'y exprime une déception spécifique quant à l'expression. Warburg avait essayé à peu près toutes les formulations philosophiques disponibles, y compris le jugement, l'aphorisme et le néologisme, sans jamais atteindre ce qu'il y a d'irréductible dans l'image (abbildlich). De ses premières hypothèses à ses dernières déclarations, il n’a cessé de rech e rcher les fonctions réelles, mentales et figuratives, dont l'image nourrit son pouvoir. Que ses réponses soient liées à l'Allemagne de la fin des années 20, cela n'atténue en rien l'intention fondamentale de modifier la relation entre l'œuvre et le spectateur qui, entre la Critique du jugement de Kant et les Leçons d'esthétique de Hegel, avait scellé d'une estampille philosophique les figures abouties de la Renaissance. Kant citait L'École d'Athènes et tout l'idéalisme allemand a fait le voyage de Dresde pour la Madone Sixtine. Hegel concluait cet achèvement à la mort de l'art. Contre l'esthétique et depuis le milieu du XIX ${ }^{\mathrm{e}}$ siècle, l'histoirede l'art de langue allemande avait protesté en inventant d'autres concepts pour d'a u tres œuvres d’art, décomptant leurs usages, destituant à la fois les canons du néoclassicisme et les obituaires des philosophes. En place de quoi Burckhardt, y a p p o rtant ses pro p res vues sur la Renaissance, avait ouvert l' his to i redela culture :

« Nous avons pris pour point de départ le seul élément invariable qui pût se prêter à une pareille étude : l'homme avec ses peines et ses œuvres, tel qưil est et sera toujours. Ainsi nos considérations auront-elles dans une certaine mesure un caractère pathologique » (Burckhardt 2001).

l'astrologie comme un témoignage de la lutte pour acquérir un espace de pensée (Denkraum)». Ce terme - que Warburg oppose à Denkmal (monument) - est plus qu'une allusion, une surenchère sur l'analyse de Alois Riegl (1984). Il prend tout son poids à un moment où « espace vital» fut un mot d'ordre politique. 
En 1927, Warburg n'avait plus à défendre ses premiers travaux universitaires où, insistant sur le trouble induit dans les belles formes par la représentation du mouvement, il avait si évidemment contredit Winckelmann sur son propre terrain. Restait à défaire une conjonction entre l'histoire et la philosophie, pétrifiée non dans la chose mais dans le concept de Renaissance. Laissant l'imitation des Anciens pour la su rvivance du paganisme, Warburg avait réinséré « ladite Renaissance » dans une réalité culturelle dont elle fut un produit exemplaire, mais instable et révolu.

Certes, à considérer la majorité des photographies, Warburg confirmait ses recherches sur la Renaissance florentine et ce qu'il avait appris de Botticelli et Ghirlandaio: l'animation des corps, une agitation des voiles et des accessoires, et tout un appareil de fruits ou de fleurs qui dérobaient ces gestes à l'interprétation narrative de la scène peinte. Dès ses premiers travaux (1893), il avait constitué ces détails en problème. Sa thèse affichait deux intentions: mettre en lu miè rece qui, de l'Antiquité, "intéressait » les artistes du Quattrocento, et tire $\mathrm{r}$ parti de cette "démonstration pour observer la constitution de l'empathie en style comme acte esthétique $»^{3}$. Touchant au pourquoi et au comment des images, le défi était déjà haut placé : faire apparaître l'empathie comme acte, en confier la formule à l' a rtiste, médiateur physique de figures où la société civile acclimate ses audaces, ses peurs et ses fêtes propitiatoires. S’il ne lui fut pas aisé de fixer les prémisses de son dernier projet, il suivait dassez près Burckhardt pour que la recherdhe de l'instant où l'art commute avec la vie, celle du moment où l'affect produit un style public, fussent conviées à relever les deux échecs de la philosophie allemande: l'expérience et la société civile.

Après cette thèse, brève et presque coléreuse, Warburg s'in té ressa aux cartons des Intermezzi, donnés à Flo rence en 1588 à l'occasion du mariage de Laurent de Médicis. Ici, l'analyse se déploie sur divers plans: séries de cartons et d'esquisses, description des vêtements et chorégraphies, succession de festivités princières et de dive rtissements populaires, et jusqu'aux livres de compte où le tissu se paie au mètre, la broderie à l'heu re de travail et les matières précieuses au cours marchand. La fête se résout dans ses dehors, ses magies propitiatoires et les ducats sonnants du prince. Et tout cela bruissait de pathos et dintérêt. Warburg ne renoncera jamais à déployer les composantes mentales de l'image, saisies dans les dimensions effectives, spatiales et temporelles, où elles se distribuent en figures, gestes et effigies, arrachées à la pulsion et à l'inconscience. Rien ici d'un psychisme des profondeurs: tout est exposé, et déjà l'attention aux détails avait relevé ce que l'on néglige de voir. Plus tard, la technique des écrans serait plus proche de celle de Poe et de La Lettre volée que de la documentation.

L'étude des Intermezzi touchait à la genèse de l'esprit public où se rencontrent peintres, commanditaires, et spectateurs. L'histoire des idées se dissipe ici comme une brume, découvrant les productions effectives et constructions mentales d'une société qui n'en finit pas de se définir. La reprise d'une gestualité au

3. Cf. "La Naissance de Vénus et le Printemps de Sandro Botticelli " (1893) (Warburg 1990a: 49). Notes de 1923, citées par Philippe-Alain Michaud (1998 : 254). 
service d'émotions vives et contrôlées, engagées dans une fonction civile et laïque, banalisait la Renaissance comme événement mais l'instituait en paradigme d'un procès de modernité réitérable, qu'il fallait comprendre dans les termes où il s'était lui-même représenté et ne cessait de le faire. Très tôt, Warburg avait limité le poids du patronage humaniste. Tout en reconnaissant à Politien la raison des figures botticcelliennes, celles du Printemps ou de la Naissance de Vénus, c'est aux peintres et aux commanditaires qu'il rend l'intelligence de leurs choix, là où se reconfigure incessamment la société civile. De l'Antiquité tardive jusqu'au Quattroento, perdurait un paganisme savant où les cartes du ciel surplombaient une vie civile interrogeant les astres et les météores, où le bestiaire cosmologique conduisait la fête que les nobles florentins s'offraient à eux-mêmes et à leurs concitoyens. Il se doublait d'un paganisme populaire, autant présent dans les cires votives, dont les familles princières encombraient la nef de Santa Croce, et dans le culte des saints que sur les almanachs vendus sur les marchés.

De quelle histoire s'agissait-il alors, et s'agissait-il prioritairement dhistoire? On a souvent rapporté la méthode de Warburg, ses curiosités et principes, à l'évolutionnisme appris durant ses années duniversité. Mais ici la philosophie de la nature tourne court. Il est vrai que The Expression of Emotions in Animals and Men l'avait libéré de la philosophie des symboles. "Enfin un livre utile", notait-il, et sans nul doute parce que Darwin touchait à ce qui lie expression, visibilité, et affect. Mais il s'agissait encore d'exp ressions faciales adhérentes et non contrôlées. La question de Warburg venait tout juste après : non pas la grimace, mais le grimage et les ajouts, en fait toute la matière des Intermezzi. En se définissant plus tard comme historien de la culture, Warburg avait franchement écarté un évolutionnisme qui aurait bien mal expliqué un phénomène de résurgence et de survie. Warburg fut inventif par déception. "Sin cè rement dégoûté de l'histoire de l'art esthétisante ", il pensa à des études de médecine. Venu à New Yo rk pour des motifs d'o rd re privé, familial, et tout autant déçu par le vide de la civilisation de l'Est de l'Amérique, il entreprit ce voyage qui le conduisit, en 1895 , vers la Smithsonian Institution, "sans oublier Franz Boas à New York " et les territoires Pueblos de l'Arizona. "J'entrpris de fuir vers les choses réelles et vers le savoir ${ }^{4}$. Ainsi Warburg décida de visiter l'Amérique de l'Ouest aussi bien dans ses créations modernes que dans ses couches profondes hispanoindiennes. Telle fut donc la bonne mesure, celle d'une histoire culturelle, et la méthode, la survivance comme archéologie.

Récapitulatif, l'Atlas reprenait donc exemplairement une question dont la résolution locale devait préparer les lemmes de la modernité. Car le système devait être assez prégnant pour capter quelque chose de l'actualité. Chaque écran (par exemple la planche 77, déployée d'une Médée en colère [Delacroix], qui serre son poignard contre son corps, à une joueuse de golf, d'un geste sacrificiel et crispé à ce bras emporté par le mouvement du club) vaut une prolepse. En 1927, 
et devant un public romain, Warburg reprit ses questions florentines. Mais il épingla bientôt sur ses écrans quelques photographies des solennités publiques accompagnant les accords du Latran, ou les derniers photogrammes du Zeppelin parus dans la presse. Si donc l'intention de Warburg ne fut pas de substituer crûment à la rationalité du jugement esthétique une généalogie venue de l'expression animale, où sera-t-elle? Les inédits, notes et lettres parlent clairement. Warburg cherche sa propre détermination sur la marge externe d'une pensée de l'image qui s'était fixée entre Platon et Kant. Il aura suffi de ses questions méthodiquement décentrées pour dénuder le socle alexandrin d'un héritage où le classicisme avait prélevé l'Apollon du Belvédére mais dont la limite fut un Laocoon emporté dans l'histoire de ses métamorphoses (Settis 1999 : chap. 1).

$\mathrm{Au}$ regard des collaborateurs de Warburg, l'Atlas serait impubliable. L'exposition de la bibliothèque He rtziana en fut une exceptionnelle ambassade. Précédée d'un fourgon de documents et bagages, elle outrepassait de mille manières les dimensions d'un livre. Le sous-titre, Mnemosyne, également gravé au linteau de la bibliothèque de Hambourg, commémorait et déplaçait un précepte platonicien. Il déclarait tout à la fois un lien matériel avec la bibliothèque, sa réalité de nouvelle Académie en face de l'Université, et une manière d'anamnèse aussi précise que la leçon socratique du Ménon, mais tirée cette fois dune tout autre géométrie, celle des gestes et des affects. L'abondant commentaire dont Warburg accompagna sa présentation, et que l'organisateur de la conférence tenta vainement dinterrompre, en appelait à la pratique de la bibliothèque où l'on peut déployer les cartes, ouvrir les dossiers d'images et consulter les livres. C'est ainsi que Warburg emportait son auditoire, et Kenneth Clark y tro uva sa vocation. Mais rien ne dit non plus que la publication - si elle fut réellement envisagée - dût se feuilleter naïvement, comme un catalogue de rep roductions. L'Atlas n'est donc pas d'abord le titre d'un livre ou le nom d'un répertoired'images, c'est un espace analytique doublé d'une opération mentale inédite. Mnemosyne n'est pas non plus un frontispice mythologique pour une collection de documents antiquaires. Renvoyant à la bibliothèque de Hambourg, elle indique un acte intellectuel non pas à propos de limage, comme le serait un jugement, ou le déchiffrement d'un symbole qui confierait à l'image autre chose qu'elle-même, mais une structure mentale dont l'image est l'ultime et explicite dépôt.

Positivement, l' Atlas valait un manifeste. Il déploie dans la manière figurative qui fut la leur, et au détriment d'intentions philosophiques compulsivement tassées sur le paradigme de la Renaissance, les figures d' exp ression et d'émotion où $s$ ' in vente la société civile. Warburg mettait au clair les termes d'une question qui s'était dérobée à l'historien de l'art et dont l'intérêt accordé par les Flo rentins à la représentation des corps en mouvement n'était que l'indice. 


\section{De la Pathosformel au Denkraum Les premiers déploiements}

En 1906, le choix d'un geste typé comme Pathosformel fut la premièredétermination du problème. Une gravure de Dürer fixait un module analytique où coïncident l'affect et le style. Au moment d' êt relapidé, Orphée est représenté le genou gauche au sol et le bras droit replié, se protégeant de sa lyre comme un guerrier de son bouclier, à la manière d'un Lapithe. Par ce choix, Dürer avait rejoint la communauté des artistes Anciens et Renaissants ${ }^{5}$. Le geste attestait la "fidélité archéologique » qui lie entre elles toutes les représentations renaissantes de la mort d'Orphée, celles-ci à quelques vases, bas-reliefs ou sarcophages antiques, et Dürer à Mantegna. Il écartait les illustrations moyenâgeuses du même topos et montrait le poète dans une gestualité noble, contrainte mais non soumise, à la mesure des émotions les plus intenses face à une mort imminente et violente. Il est notable que Warburg ait pris pour paradigme du Pathosformel ce quasi-autoportrait de Dürer en Orphée, plutôt que son réel autoportrait en Schmerzmann. Non plus une attitude prise d'une station de la Passion, épre uve d'une humanité bafouée, mais le geste où Orphée se défend de sa lyre, jouant de son immortalité de poète contre sa mortalité humaine. En Dürer, le choix du paganisme antique se fit dans le mouvement d'une réflexion sur la Renaissance menée à partir de l'Allemagne et affranchie de la dire ctive humaniste et néoplatonisante. Dürer arbitrait entre le (nouveau) paganisme romain et cette autre Renaissance que voulut être la Réforme, et Warburg saisit une opération consciente et critique à même le travail du peintre.

Cette Pathosformel fut la première et décisive réélaboration de la scène botticcellienne initiale, Warburg y appliquait ce qu'il avait appris entre temps des Intermezzi et du paganisme amérindien. Il suivra Dürer jusqu'au moment aporétique de Mélancolie I. Partagée entre la géométrie del'équer reet du compas et les monstres astrologiques, la gravure contrariait le savant équilibre du Moderne et de l'Antique imaginé à Flo rence. Le processus d'image avait pris possession de lui-même, et le peintre se trou vait maintenant impliqué dans un développement de connaissances ordonnateur des cérémonies humaines, complice ou dénonciateur de leur cosmologie mentale. La Pathosformel saisit la configuration de l'affect en style public, où chacun sera convié à penser la gestualité comme un habit de fête ou de deuil, moins oppressant et plus magique qu'un choix de vie. Les épisodes de la vie de saint François, se dévêtissant et se revêtissant de bure, endossant la civilité d'un ord re mendiant confirmé d'une règle papale, sont deux et trois fois emblématiques. Ils le sont pour Ghirlandaio, le peintre de scènes sacrées et de scènes civiles, pour la famille Sassetti honorant son saint pat ron, et pour Warburg qui en inscrit le rituel dans le procès même de la figuration comme incessante refiguration. Cette formule pathique en son vocable même était encore très proche d'un modèle discursif sur lequel Warburg n'a

5. Pathosformel apparaît en 1905 dans Dürer et l'Antiquité italienne. Voir Moshe Barasch 1991. C'est beaucoup plus tard, en 1924, que Warburg s'identifie lui-même comme historien de la culture. 
cessé de travailler tout en en prenant distance. Sur l'instant, elle défiait l'opposition stoïcienne entre pathos et ethos (Settis 1997 : n. 61) mais aussi apportait un exact et subversif parallèle à ces formules de la moralité où Kant avait uni, dans lincertain montage d'un impératif catégorique, un stoïcisme dégrisé de son délire cosmologique et l'infigurable monde des fins ${ }^{6}$. En 1893, le détail des vêtements agités avait été le support occasionnel d'une question qui touche à la raison d'êt re de l'image. Le schème de la Nymphe, que Warburg abrégeait dans ses dernières notes manuscrites comme l'élément simple d'une formule chimique ( $N y$ ), illustrait aussi une transformation que le Nachleben - formule d'historien - ne capturait pas exactement et qui réclamait son droit. Au retour du voyage en Californie, l'histoireavait cédé au procès même de la culture, où Cu rtius avait bientôt suivi Warburg. L'image, la plus précise, accepte, comme la mort/immortalité d'Orphée, le retournement du sens et, comme la Nymphe, une suite infinie de modifications et de polarités que la photographie saurait fairevoir en multipliant les comparaisons.

Les écrans de Mnemosyne empruntaient à une pratique déjà familière. Trente ans plus tôt, au retour de son séjour de quelques semaines en pays hopi, Warburg ava it accompagné son récit devant le Club américain de Hambourg, puis la Freie photographische Vereinigung de Berlin, par la projection d'une trentaine de photographies dont il était, pour beaucoup d'entre elles, l'auteur. Dans la suite, ses conférences d'histoire de l'a rt furent illustrées de diapositives. L'usage d'épingler des reproductions sur des écrans mobiles était l'apport de Saxl, inventé quand il fut quelques mois durant instructeur aux armées. On sait que Warburg a préparé quelques expositions pédagogiques. Soucieux de donner les techniques les plus modernes à sa bibliothèque scientifique, il n'a pas manqué d'institutionnaliser ces pratiques sur lesquelles on ne reviendrait pas. On pourrait donc vouloir tracer une ligne droite, illustrative et documentaire, allant des premières conférences jusqu'à la présentation de l'Atlas à la bibliothèque He rtziana. Mais d'une circonstance à l'autre, technique, corpus d'images et argument, tout avait changé. En 1896, Warburg apportait le témoignage de photographies et de dessins indigènes qui captaient l'en deçà de la civilisation moderne et pouvaient servir un propos évolutionniste : " un coin de nature vu par un Kodak ", disait-il (en français), à moins qu'en parodiant un mot de Zola il n’ait substitué l'opérateur Kodak à ce «tempérament » dont se réclamait le naturalisme hautement pathique de Zola (Steinberg 1995). Dans ces mêmes années, Warburg avait aidé à l'acquisition de poteries hopis par le musée ethnographique de Berlin. Ces photographies et croquis ont été de nouveau montrés en 1924, accompagnant la conférence donnée par Warburg à Kreutzlingen, après quelques années d'un désarroi mental consécutif à l'épre u ve de la guerre. Elles s’y trouvaient comparées à dautres, prises du répertoire classique. Entreprise modeste, au regard de ce millier de photographies venues de sources encore plus dissemblables qui articuleront les écrans de l'Atlas. Néanmoins l'ordre des thèmes en donnait un état préalable, modeste et décisif. Warburg était parfai- 
tement conscient de la solidité d'un exposé qui lui fit solliciter l'épre u ve, convaincre Binswanger de sa guérison et communiquer son texte à Cassirer ${ }^{7}$. La construction se fait en trois temps.

- Des images amérindiennes, Warburg mit en avant l'élément "rationnel " (c'est-à-dire architectonique) : la structure des maisons pueblos, dont l'ordonnance verticale, de la kiwa au grenier, privilégie un vecteur cosmologique. Le schème en est répliqué sur des tissages, poteries et principalement sur l'autel abrité dans la kiwa. Puis il décrit trois danses selon une complexité croissante. D'abord le mimétisme animal de la Danse de l'antilope, puis une danse à masque (Humiskatcina) sollicitant l'eau et le maïs. Elle est accompagnée d'un mime sexuel bouffon - intermède satyrique dit Warburg. Vient enfin un ample cérémonial, conduit sur plusieurs jours et dont les protagonistes sont une variété de serpents venimeux, apprivoisés et drogués pour la circonstance. Ici la magie mimétique et l'injonction propitiatoire cédaient aux phases d'un rituel réglant dans l'espace et le temps l'économie agraire des Hopis, leurs initiations, le calendrier des récoltes et des saisons, le tout reporté sur les mailles d'une cosmologie. Simultanément, Warburg soulignait l'objectivation du rite dans un schème épuré. Le serpent, figure de vie et de renaissance, est repris dans le signe de l'éclair et de la pluie bienfaitrice. Représenté par un zigzag, il se fait échelle du ciel et figure stellaire. Ce long rituel, au terme duquel les serpents sont rendus au désert, exclut le sacrifice. Warburg le décrit comme un pôle de "paganisme absolu ".

- Suit une comparaison avec le paganisme antique et ses survivances, au Moyen Âge et à la Renaissance. Le thème du serpent fait charnière - " le serpent est un symbole international pour répondre à la question : d'où vient la destruction, la mort et la douleur qui sévissent dans le monde ? " Mais il n’y parvient si bien que pour être aussi un emblème de résurrection. Les Pueblos auraient-ils su apprivoiser ou négocier la mort ? Comme l'Orphée de Dürer? Le serpent est l'opérateur d'une inversion pour être d'abord son propre antidote. Ainsi, sur un manuscrit de la fin du Moyen Âge, dans un assemblage comportant les images ambiguës d'Esculape au caducée, une citation de l'apôtre Paul, et le serpent d'airain que Moïse, dit-on, commanda pour éloigner les serpents réels, on voit un serpent crucifié, accolé à une Passion ${ }^{8}$, et tout cela juxtaposé comme par collage sur la même page du Speculum humanae salvationis. Le Laocoon vient enfin, cette fois retiré au catalogue de l'éthos stoïcien - à moins que l'ethos stoïcien n'ait de ce fait retrouvé sa place dans les figures du paganisme.

Ainsi, plutôt que de s'en tenir à la récurrence iconographique d'une image obsessionnellement présente dans le psychisme humain, et si immédiatement phallique comme il l'a vait lui-même noté, Warburg suit le dédoublement du

7. Publié en anglais par les soins de Gertrud Bing (Warburg 1938-1939) dans The Journal of the Warburg Institute. Édition critique allemande Schlangenritual. Ein Reisebericht (1988). La traduction française est annoncée.

8. Cf. Warburg 1988 : fig. 8, Kosmologisches Ornament an der Wand der Kirche von Ancona ; fig. 26, Eherne Schlange und Kreuzigung, aus Speculum humanae salvationis, British Library (London), Add. MS 31303. 
schème du serpent et ses significations inversées. En Grèce, enroulé sur le bâton d'Esculape, il se fait guérisseur. À Alexandrie, il fait partie du bestiaire astrologique. À Jérusalem, le serpent d'airain fut apotropaique de sa prop re menace. Plus tard le schème est scindé entre le bois de la croix et la figure salvatrice de l'homme crucifié. Et toujours, l'effigie est magique par l'efficace immédiate qu'on en attend, et symbole cosmique par son élévation, son association aux figures astrales ou aux météores, au salut du monde, et à la recherche de causalités. Quelques années plus tôt, Warburg avait montré comment l'astrologie alexandrine imposait ses prédictions aux Réformateurs ${ }^{9}$. Si donc la permanence du paganisme, sa capacité à nourrir la médiation culturelle par les images, et l' i ntelligence architectonique qu'il déploie, l'avaient emporté sur l'imitation de l'Antique, c'est qu'elles en donnaient la clé. Warburg laissait ici une iconologie amérindienne qui l'eût vraisemblablement conduit au cycle du serpent à plume, à suivre sa brève description de motifs relevés sur les poteries hopis. Le schème du serpent le retient au titre de forme symbolique. Il mériterait, dit-il, un nouveau chapitre dans la philosophie (néokantienne) du als ob. En fait, il avait rejoint fonctionnellement le catalogue des Pathosformeln: à pre u ve qu'il se prêtait aux significations inverses de salut et de péril, comme le geste du jeune David triomphant rep renait celui, de terreur et de protection, du pédagogue des Niobides dont il retournait le sens - un tour vraisemblablement comparable à l'amphibologie de gift (don) / gift (poison) signalée par Mauss.

Certes, Warburg faisait sa place à l'évolutionnisme, présent dit-il dans l'appariement totémique des hommes et des espèces. Mais il s'y tro u ve dépossédé de tout déterminisme dès lors que la déclaration d'une ancestralité animale est un choix culturel. Les apparentements totémiques sont argumentés, soutenus par des mythes et des rites animaliers qui sont comme des affinités électives, fondées sur une préférence qualitative. Ainsi la force de l'ours qui n'est que force, ou la rapidité de l'antilope qui est la course à l'état $\operatorname{pur}^{10}$. Bien que formulé dans une conceptualité durkheimienne, c'est un choix qui repose sur un jeu adjectif, sur une intelligence du superlatif comme forme absolue et principe de la nomination qualitative ${ }^{11}$. Cette remarque rejoint l'exagération des mouvements, relevée sur les figures hellénistiques et renaissantes, que Warburg comparait à des suffixes grammaticaux. Il n'abandonnera jamais un parallèle entre les tournures linguistiques innovantes et les formules iconographiques. Quant à la théorie

9. Cf. Warburg 1990b : 250 ; à propos de la démonologie astrologique : «en tant que signes des planètes, ils élargissent l'espace, offrant des points de repère à la migration des âmes dans le cosmos, en tant qu'images des planètes, c'étaient en même temps des idoles que la créature misérable cherchait à rejoindre dans une union mystique ". Ici, la figuration est clairement rapportée à ses deux composantes, de cosmologie et d'empathie.

10. Warburg 1938-1939: 283. Walter Benjamin avait publié un long article sur Les Affinités électives de Goethe, dont il avait relevé les éléments mythiques. L'article parut en 1922, dans la revue de Hoffmannsthal, Neue deutsche Beiträge, que pratiquait Warburg. Qu'il suffise ici de renvoyer également à Claude Lévi-Strauss (1962).

11. Voir une note analysant en termes de qualités la forme symbolique du serpent, publiée par Gertrud Bing: "Welche Eigenschaften bringt die Schlange mit, um sich als verdrängender Vergleicher in Literatur und Kunst einzustellen?" (Warburg 1988: 59, n. 4). 
darwinienne des émotions, elle avait depuis longtemps fait place aux pratiques civiles du masque et du portrait de cire (Warburg 1990c: 124). L'attention au détail, ici le motif du serpent, ouvrait soudain sur ce qui n'en est pas un. L'histoire des religions vient expliquer ce que la survivance iconographique ne dit pas. Quand Warburg compare le paganisme amérindien avec le paganisme savant du monde hellénistique, il ne cherche pas une origine. Il montre, comme sur un cas géométrique simple, comment s'articule sa prop re démonstration. "L'indien se tient à mi-chemin entre le magique et le logique, et son instrument d'orientation est le symbole."

Plus tard, Warburg minimisa l'intérêt de cette conférence, arguant que ni ses sou venirs, ni le petit nombre des documents photographiés, ni la brièveté du temps passé chez les Indiens pueblos, ne lui permettaient de prétendre à un véritable travail scientifique. La réserve porte à juste titre sur les limites de son expérience de terrain et sur un exposé qui ne pouvait éviter d'ap procher le « récit de voyage "; elle porte tout autant sur le thème, ou mythème du serpent. L'Atlas n'en retiendra que la fonction. Il avait suffi que Warburg mît en série quelques images prises du Moyen Âge allemand et des premiers temps de la Renaissance pour que le paganisme lui permît de déplier un événement qui ne fut pas limité à Florence. Il lui donnait dabord ses axes géographiques: du nord au sud, autant que de l'est vers l'ouest. De plus, en incluantl'apport des pays nordiques et de la Réforme dans les abords de la Renaissance florentine, il en étirait l'instance sur une temporalité beaucoup plus lente, plus réelle historiquement et traversée d'échanges, de choix, et plus proche d'une archéologie que d'un récit. Enfin, il faisait apparaître un jeu de tendances contradictoires entre deux pôles, la fonction magique du sacrifice et la contemplation cosmique. Warburg avait déjà montré l'ambiguïté des cérémonies civiles et religieuses de la société florentine, engouée de mannequins perpétuant la présence des ancêtres. Cette fois, les poupées katchinas suspendues dans les maisons de terre des Pueblos étaient comparées aux crucifix accrochés dans les foyers chrétiens. Ou encore, la description des initiés descendant un à un vers la kiwa en prenant appui sur les encoches d'un poteau taillé à la manière d'une échelle cosmique, coïncidait symétriquement avec l'ordonnancement de la famille Sassetti entrant par un escalier ascendant dans la fresque franciscaine de sa chapelle vo tive ${ }^{12}$. Ici comme là, l'état civil y acquiert sa règle séculière en traversant des espaces positivement incompatibles. En t rel' a rt et la philosophie de la Renaissance, Warburg aperçoit le palimpseste anthropologique des peurs, des initiations et des intérêts. La confrontation d'images hétérogènes, pour lesquelles n'existaient encore aucun musée et aucune collection, qui n'a vaient aucune unité ni de lieu ni de temps ni de style, et toutes recadrées dans l'unique médium de la photographie, avait modifié profondément le genre de la conférence accompagnée de projections. Maintenant Warburg affinait son point de comparaison: nullement la gestualité, la danse et le masque, mais dabord le paganisme et son régime

12. Warburg 1990c: 112 et 113 et la planche 10 de l'Atlas: Domenico Ghirlandaio, La Confirmation de la règle de l'ordre de saint François, fresque de la chapelle Sassetti, dans l'église Santa Trinita. 
dinteressions corporelles, où les rites sont indissociables de leur concrétion en symboles. Sur les murs de l'église d'Ancona, Warburg avait photographié un diagramme en escalier, répliquant dans l'enceinte du temple chrétien le schème cosmologique païen de l'échelle et de l'éclair comme un flagrant exemple de la survie du paganisme. Mais à considérer ces fresques, les décors des poteries, les schémas de son informateur, ou les dessins d'enfants tracés sur une feuille de cahier d'écolier qui avaient survécu à deux acculturations chrétiennes, l'une catholique et espagnole, l'autre protestante et américaine, il parlait aussi justement d'une Renaissance hopi.

- En conclusion, Warburg posait une question : faut-il tuer le serpent comme y incite la civilisation américaine technicienne et sanitaire, plutôt que de s'en approprier la médiation? L'occasion en était la photographie d'un bourgeois de San Francisco portant chapeau haut de forme et redingote noire, que l'apparal avait saisi s'avancant d'un pas assuré vers l'objectif, tournant le dos à un poteau chargé de fils électriques. Ici aucune figure d'interession, l'énergie répond instantanément à la commande. Mais Warburg n'en montrait-il pas déjà plus dans ce montage photographique involontaire? Sur la gauche apparaissent une coupole néoclassique et une façade à colonnades, insinuant leur présence tutélaire dans le dos du Yankee qui semble tout ignorer du décor civil devant lequel il s' a vance mais dont - église, banque ou palais de justice - il tient, à n'en pas douter, toute son assurance. Ce paganisme, affiché et dénié, sera la substance et le ressort del'Atlas. On ne tue pas une forme symbolique, on traque ses métamorphoses.

\section{Les planches alphabétiques}

«Est-ce que l'intuition et la logique sont des partenaires égaux dans la production de l'œuvre d'art?» (Warburg 1988 ; Forster 1996). Touchant aux raisons d'êt re de l'image, Warburg écartait une esthétique conçue pour l'éducation ou le plaisir pur du spectateur, si aisément et illusoirement conduit de la peintu re à ce qu'elle montre. Entendue littéralement, sa question disait plus, touchant dans ses termes mêmes au nœud du jugement de goût kantien. L'ultime support d'un écran parsemé de photographies faisait plus qu'ajouter un dernier chaînon dans une succession de fresques, cassone, tapisseries, images dÉpinal, tableaux de chevalets, plafonds, galeries et musées - ces Stoa Poikilé des temps modernes - qui tous perpétuaient et transformaient ce "paganisme» dont on comprend maintenant qu'il n'était plus pour Warburg un thème de liconologie antique mais le ressort de la figuration. En éteignant délibérément tous les prestiges de la représentation dans le monochrome d'une reproduction photographique, Warburg extorquait à l'image ce que sa fascination auratique dissimule. Mais il fallut que l'Atlas ait résolu singulièrement un problème général, énoncé par Benjamin quand il traita du Trauerspiel. «C'est le caractère de tout écrit philosophique qu'il ne peut éviter d'affronter la question de son mode dexposition (Darstellung)" (Benjamin 1928 : 207). Dans les deux cas, la réponse fut de faire servir les dimensions de sa propre exposition (Darstellung) pour capturer les 


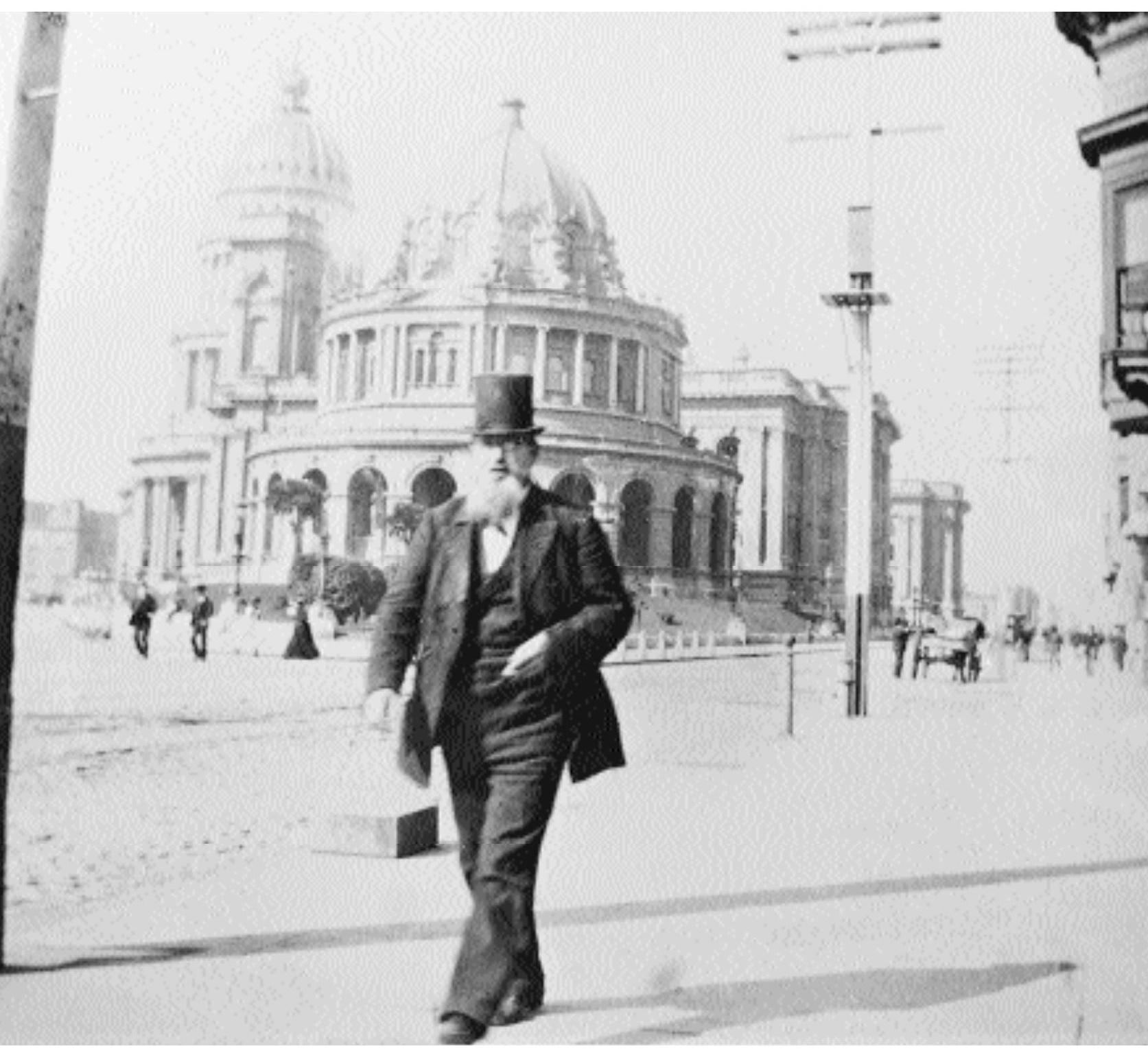

Oncle Sam (cl.Warburg Institute) 
dimensions - autres - de ce dont il est traité. C'est-à-dire d'en finir avec la vieille technique rhétorique d'une introduction naturelle in medias res. Confirmant cette rupture d’avec la phénoménologie de l'expérience, sa stratégie initiatique et pro treptique, Warburg redéployait le jeu de la représentation (Vorstellung) sur un Atlas de géographie humaine et de cartes mentales.

Cette fois, la simple poursuite d'un thème, qui avait été la solution de fortune pour l'exposé de Kreutzlingen, n'y suffirait pas. Warburg affronterait cela même sur quoi il avait dramatiquement trébuché durant la guerre et l'immédiat aprèsguerre, vaincu par tant de représentations, informations et photos de presse fébrilement amassées, surchargées d'émotion ou soupçonnées de propagande. "La guerre de 1914-1918 m’avait imposé la vérité dévastatrice que l'homme, rendu aux forces élémentaires, sans aucun lien, est le maître invincible de ce monde $»^{13}$. L' Atlas trame les liens d'une économie mentale d'après le sacrifice humain dont Warburg avait perçu la nouvelle ivresse. Et c'est cela, ce sacrifice humain enfoui dans ses rites substitutifs, qu'il appelle le paganisme. Que la solidarité soit évidente entre l'agencement des écrans et la propre catharsis de Warburg, on ne saurait ni le masquer ni le faire valoir contre sa démonstration. Le point est ailleurs. Si Warburg ne fut pas américaniste et s'en défendit, comme le note Kurt Forster, il était entré dans l'ethnographie de son propre pays, laquelle sollicite autant que l'ethnographie lointaine un terrain dobservation et une conscience singulière, dont l'absence invaliderait le travail de description. Le long commerce des images, et l'abandon des concepts où il avait été éduqué, lui en avaient donné les moyens. On ne s'étonnera donc pas que les documents amérindiens ne figurent pas dans l'Atlas : ils avaient donné à Warburg la première ébauche de ses prémisses. Jouant maintenant sur les composantes de la figuration, le geste émotionnel typé et la sous-figuration cosmologique impliquée, Warburg allait transformer en hypothèse constitutive ce qu'il n'a cessé de décrirecomme une schizophrénie interne à l'image, une tension entre sa magie et son architectonique. La solution proposée appelait un préambule, les planches alphabétiques en ont fixé l'essentiel.

Planche A. - Y sont montrées, de haut en bas, une carte du ciel du XVII siècle, une carte de géographie historique où se tro u ve reportée la diffusion de l'astro logie alexandrine autour du bassin méditerranéen jusque dans l'Allemagne du No rd, enfin l'arbre généalogique de trois générations de Tornabuoni, la famille florentine qui fut le conscient mécène des figurations à l'Antique dans l' Italie du Qiattrocento. Trois cartes pour montrer sous différentes projections ces enveloppes de monde et dhistoireoù le comportement des hommes prend figure.

Le ciel historié, épinglé au registre supérieur, n'est ni ce que l'on voit ni un lieu d'où l'on voit, mais une carte comme les autres. C'est aussi la restitution de l'image comme ébauche d'un diagramme cosmologique, une arche dalliance entreles dieux et les hommes, un pari sur la nature, cette fois reporté sur une cartographie hellénistique. La figuration du ciel, comme une rotonde mise à 

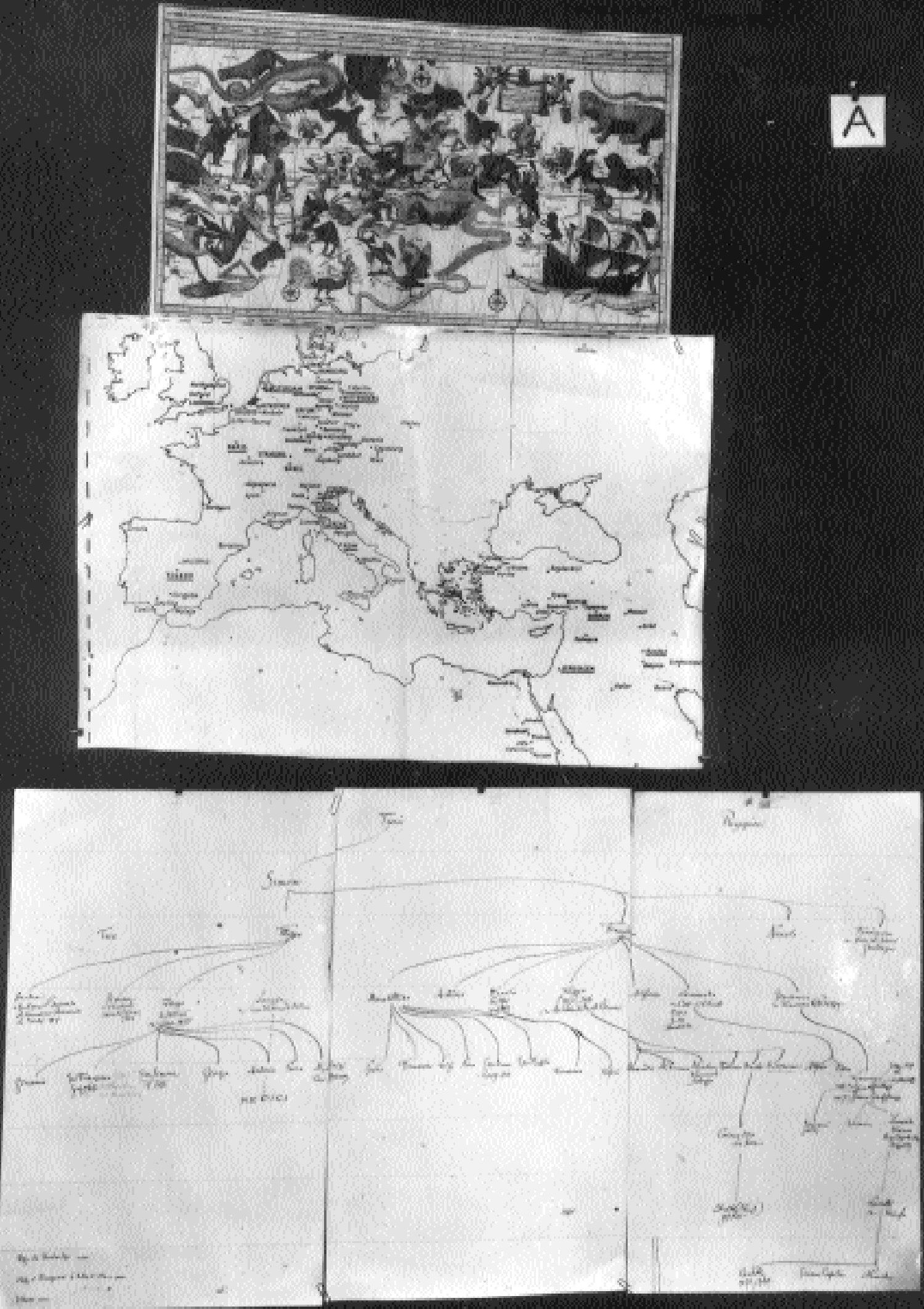
plat, garde quelque chose du bouclier d'Achille, ce premier miroir du monde. Mais surtout, tout occupée de figures zoomorphes accompagnant les constellations astrales, cette carte matérialise, à la première page de l'Atlas, son impératif: Per monstra ad astra. "Toute la tragédie prométhéenne de l'homme tient en ceci qu'il n'y a pas de ciel fixe au-dessus de nous ", ce dont Georges Bataille, moins de dix ans plus tard fit le thème d'un roman ${ }^{14}$.

La seconde carte répertorie les villes par où a transité l'astrologie alexandrine, de l'Antiquité tard ive à la Renaissance et jusqu'à Luther. Sur ce postulat d’une histoire culturelle, les images et les manuscrits qui la diffusent ont voyagé du sud vers le nord, et d'est en ouest :

« Nous sommes au siècle de Faust, où le savant moderne - à mi-chemin entre la pratique magique et la cosmologie mathématique - cherchait à conquérir l'espace de la réflexion (Denkraum der Besonnenheit) entre l'objet et lui. Car il faut sans cesse reconquérir Athènes depuis Alexandrie »(Warburg 1990b [1920]).

Cette Athènes-là, tout juste un point noirci sur la carte, est une " Athènes engloutie (verschobene)", dont Alexandrie figure le pôle opposé ${ }^{15}$. S'y explicite une tension interne à la mémoire culturelle qui rappelle l'opposition entre Apollon et Dionysos. Vînt-elle de Nietzsche, cette fiction philosophique trouve son lieu propre dans une production d'images tout entière distribuée sur ses supports, prise dans la séquence de sa laïcisation, et dont Warburg promet de faire voir les déterminations immanentes.

Le propos, tout juste circonscrit à l'ère méditerranéenne, se focalise en bas sur une séquence florentine. Le premier écran s'achève ainsi avec ce point d'histoire sociale. La vie civile est cela même que les peintres, médiateurs inspirés de l'Antique, eurent pour fonction de projeter sur cette scène de gloire et de monde où s'apprivoisent les monstres. Warburg avait depuis bien longtemps opposé à la sensibilité du Moyen Âge cette manière florentine d'instituer la jouissance de la vie civile en en confiant l'image au patronage de quelques saints intercesseurs. Quelque chose avait commencé qui ne s'achèverait pas, incitation qui s'entretient elle-même, comparable en cela au plaisir sans but externe dont traitait l'esthétique de Kant (Gombrich 1970 : 157). L’Atlas lui donnait sa concrétude et ses chances modernes.

Planche B. - Ouvrant sur la figure humaine, ses peurs et ses magies conjuratoires, elle confronte neuf dessins, représentant autant de manières dinscrire le microcosme humain dans le macrocosme. Ainsi les figures du zodiaque projetées sur le corps mythique d'Hercule, ou même directement écrites sur cette anatomie

14. Cf. A Warburg 1990b [1920]. Également, mais pour une expression apocalyptique et politique du même sentiment, cf. Georges Bataille (1952 : page finale), où le ciel s'effondre sur la terre boueuse d'un cimetière. Les veilleuses déposées sur les tombes en sont les funèbres étoiles. Ce livre, commencé en 1936, fut publié après la guerre.

15. Laissons ici cette vision déconcertante d'Alexandrie. Elle corrobore l'exaltation du classicisme athénien qui fut le point d'honneur de l'université allemande. Alexandrie est, dans l'argument de Warburg, un pôle idéal dont il ne faut pas solliciter l'identité à un moment donné de l'histoire. 

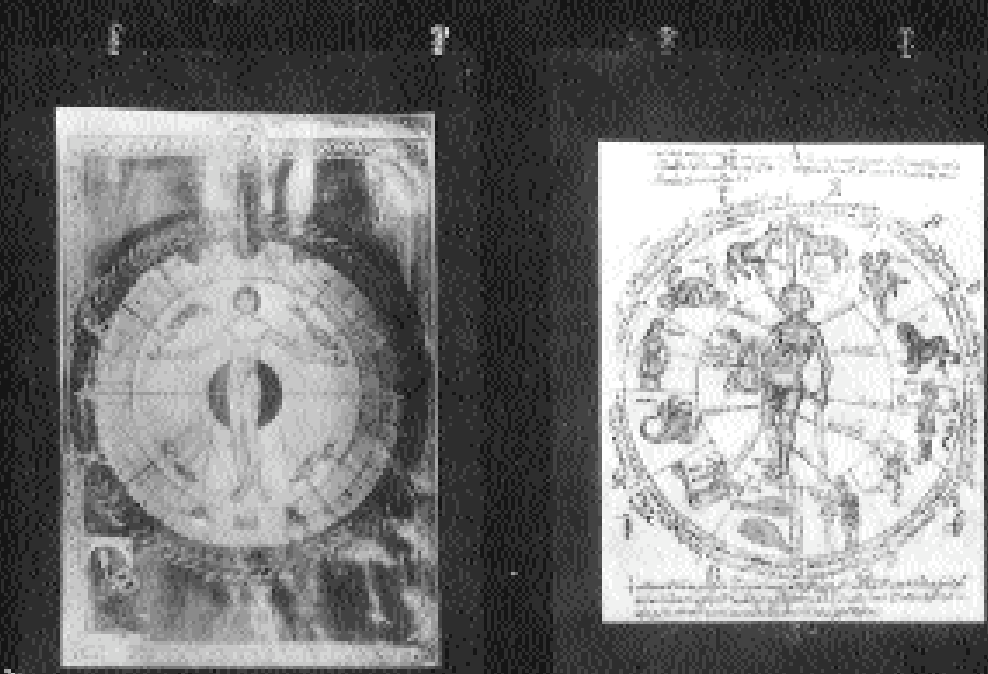

$y$
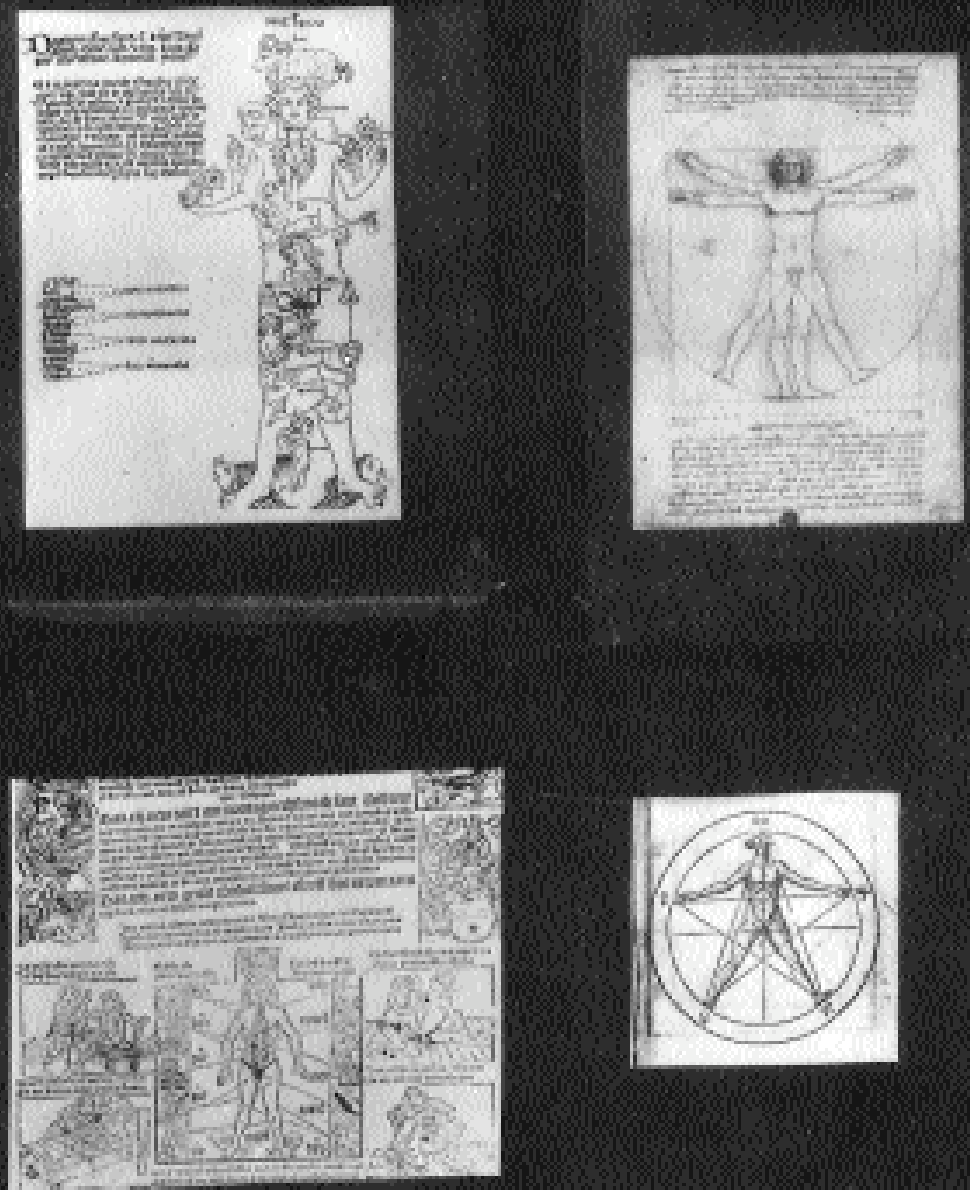
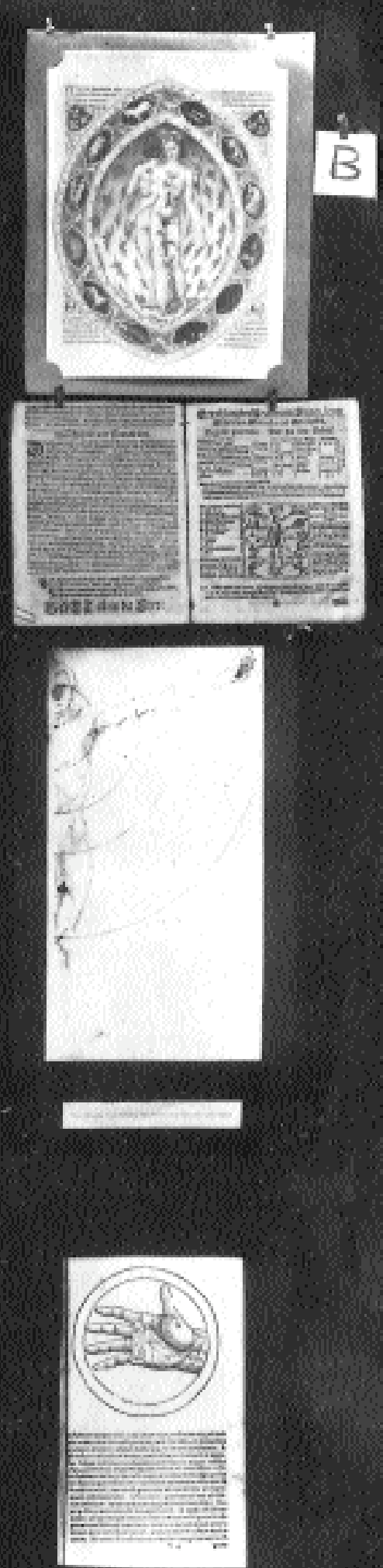
rudimentaire, tel un tatouage. Ces points astrologiques furent aussi des repères pour la saignée, confirmant le lien entre les matematici - c'est-à-direles astrologues - et les medici qui ont transmis le paganisme sous le couve rt de leur profession (Warburg 1990b: 262). Deux figures, dues à Vinci et à Dürer, sont directement tirées du prototype de Vitruve. Un homme inscrit tel un X dans un cercle parfait est la mathématisation idéale d'une anatomie masculine, un instant oublieuse des orbites astrales dont elle tient son géométral et du corps réel qu'illustrent trop bien les caricatures des mêmes Vinci et Dürer. Schème venu droit de l'Antiquité païenne, c'est aussi la variante d'une mise en croix sans bois ni clous, ce que serait l'h eu reuse inscription d'un homme dans l'orbe parfait d'un univers épuré de ses démons. Sur ces dessins renaissants, et comme par un ultime traveling de l'objectif, le microcosme se voit seul et le macrocosme n'est que la ligne géométrique, cercle, carré ou mandorle, qui le circonscrit. L'humanisme y est serein et achevé. Usant des mêmes circonscriptions et inscriptions, l'hypothèse copernicienne du kantisme apparaît, de par la seule évidence des tracés, en être un cas tardif et part iculi i er ${ }^{16}$. L'écran s' a c h è ve sur deux figures magico-mystiques d' un corps zodiacal à l'écritu reraffinée. L'une provient du Livre d'heures du duc de Berry et l'autred'un manuscrit de Hildegardde Bingen.

Tout le malheur de l'homme, notait Warburg, commence avec le vêtement. Faut-il comprend re: avec la peur (dont on dit qu'elle colle à la peau) et sa magie conjuratoire, avec le premier tatouage et la première vêture, avec les premiers outils et les premières figurations? Cette iconologie intermédiaire (Ikonologie des Zueischenraum), oscillant entre l'assimilation magique et les figures cosmologiques, comme de successives protections, criblera d'images les écrans de l'Atlas ${ }^{17}$. Depuis ses recherches sur les attributs du corps en mouvement, l'agitation des voiles, des chevelures postiches et des accessoires, tout comme dans sa description des danses pueblos, avec masques, pectoraux et crécelles, et maintenant dans ce parcours entre le tatouage ziodacal et le corps olympien de Vitruve, Warburg insinuait tout un espace d'images entre la beauté adhérente de l'animal et la beauté libre comme production de la nature ou de l'art. Il s'agit de ces images que Kant avait plus qu'ignorées, explicitement condamnées, n’y voyant que des fioritures "comme font les Néo-Zélandais avec leurs tatouages " (Kant 1965 [1790]: $\$ 74$.$) .$

Planche C. - Au registre supérieur, on voit deux figurations du système du monde, dues à Kepler. Elles seront retenues pour l'exposition Sternglaube und Stemekunde, que Warburg préparait dans ses derniers jours. La première représente le système solaire par un emboîtement de solides réguliers. L'autre est un

16. On comparera à ces figurations la distribution circulaire en quatre points fixant successivement les fonctions du jugement, et la description qu’en donne Kant (1967 [1781] : \$ 39) : "C'est toujours le même fil conducteur qui, comme il doit toujours être conduit par les mêmes points fixes, déterminés a priori dans l'entendement humain, constitue constamment un cercle fermé. » Le diagramme logique de la subjectivité transcendantale, cas limite, réduit le sujet inscrit au point central où il a perdu toute substance, il n'est que sa position et sa fonction.

17. Cf. A. Warburg, Journal, juillet 1929, cité par Gombrich 1970: 253. 

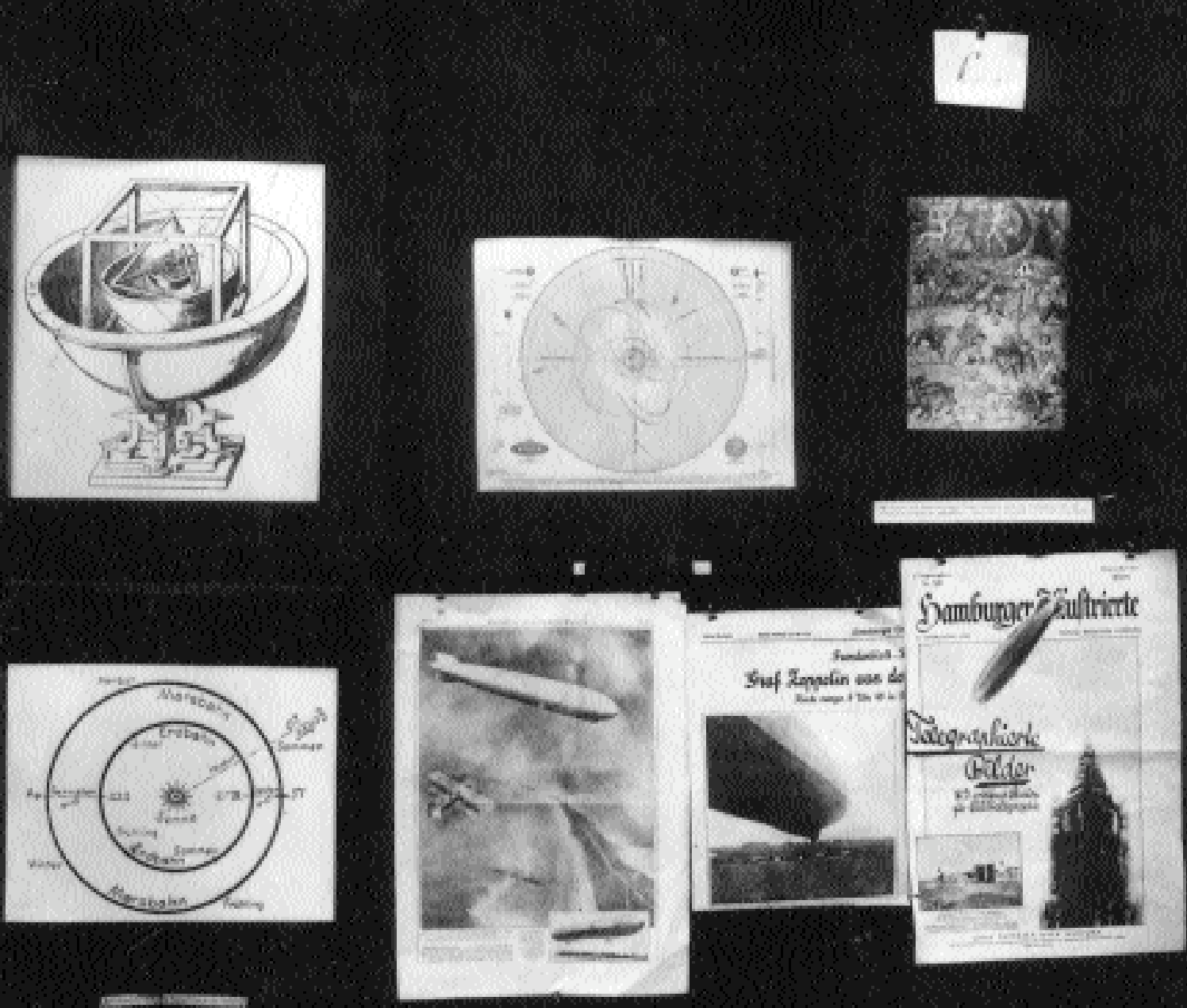
tracé planaire de l'orbite elliptique de Mars. C'est un hommage à Cassirer, qui voyait dans l'ellipse képlérienne la première forme symbolique, et comme une citation du même transcrite dans les termes de l' Atlas. Ce patronage ouvrait une cartographie inédite fondée sur une continuité entre la forme symbolique et le Pathosformel, entre le post-kantisme et l'intelligence d'un paganisme que Kant avait si violemment rejeté, coupant net entre Euclide, Copernic et les cosmologies du visionnaire ${ }^{18}$. Le Denkraum de la bibliothèque et le Zwischenraum des images s'y trouvaient en conjugaison.

Ce pas franchi, il ouvrait un droit de suite, ainsi pour les deux images fixées au bas de l'écran, tirées de la presse d'actualité : la trajectoire plongeante d'un Zeppelin vraisemblablement près d'atterrir sur la côte américaine et la même, traversée d'une inscription en écharpe, telegraphierte Photographie. Cette photographie d'un dirigeable en forme de cigare, nef aérienne encore massive, pourrait être la première d'une série continuée jusqu'à l'envol d'un avion supersonique. $\mathrm{Ne}$ pas oublier que Kafka et Wittgenstein se sont déplacés pour assister à une démonstration d'aéronautique ${ }^{19}$. Mais surtout, dernière venue sur ces planches introductives qui extraient des images leurs formules d'émotion, elle résout presque point par point la photographie de cet Américain de San Francisco que l'objectif avait saisi sur le double fond d'une coupole néoclassique et d'un poteau télégraphique. Le dirigeable, coupole et nef, tourne à son profit l'énergie gravitationnelle et la force des vents. Warburg s'était particulièrement réjoui de savoir qu'une manœuvre habile, due à des instruments de mesure perfectionnés, capteurs et émetteurs, avaient permis à l'équipage du Zeppelin d'éviter une zone de turbulences météorologiques.

Comme Cassirer avait voulu conjurer la clôture des concepts et formules d'expérience par les formes symboliques, Warburg y ajoutait son Atlasd'images. Avec cette singularité qu'aucune image ne s' exempte de la chaîne à laquelle elle appartient, ni n'arrête la suite de ses métamorphoses. Seule leur succession fait apparaître une continuité archéologique qui les anime comme un Kunstwollen, lequel ne produit ni la figure nette d'un monde ni ne la contredit. L'histoire de l'émotion est une histoire qui n'en finit pas. Ses formules occupent la limite visible de la conscience, crête mouvante d'une production incessante dimages qui l'explicitent si elles ne la conjurent.

\section{Mnemosyne}

Ces trois prémisses alphabétiques avaient donc exempté la photographie de sa fonction primaire de reportage. "L'Amérique m'a appris la photographie ", disait Warburg ${ }^{20}$. Mais que lui avait appris la photographie, qui ne lui serait pas

18. Voir, après Histoire universelle de la nature et théorie du ciel (1755), puis en 1764 les Réflexions sur les maladies de la tête et Observations sur le sentiment du beau et du sublime, où Kant traite de la mélancolie, enfin, pour clore ces écrits précritiques, Les Rêves d'un visionnaire tirés des rêves de la métaphysique (1768). 19. Voir le court récit de Kafka, «Les Aéroplanes à Brescia " (1980 [1912]).

20. Sur l'importance du voyage aux États-Unis pour la découverte des procédés photo-cinématographiques, voir le livre indispensable de Philippe-Alain Michaud (1998). 
clair đemblée? Comment fut-elle l'opérateur sous l'effet duquel une brève expérience amérindienne et ses pro p res questions européennes étaient entrées en commerce, et comment serait-elle le truchement d'une anamnèse?

Cette austère distribution de clichés de petit format, serrés sur un fond de toile noire, jouait sur l'étonnante capacité des images à transiter d'un support à un autre: arcs de triomphe, statues en ronde bosse, bas-reliefs, folioles de propagande, tapisseries, fresques, peintures de chevalet, manuscrits, cartes, et maintenant "rep roductions mécaniques». Ce même transfert atteste qu'une image prégnante impose son schème - tels le Zeppelin ou la joueuse de golfe - sous le couvert de qualités esthétiques ou d'une histoire (storia) dont elle se sert plus qu'elle ne la sert. Et ce fait révèle quelque chose d'une opération inconsciente, entr'aperçue sous les notions de sunie, imitation ou renaissance. La formule pathique, comme Warburg le montrait à propos de Düre r, passe l'épreuve d'un transfert critique, choisi et répliqué comme un mythème lévi-straussien. Ce en quoi elle acquiert ou démontre une fonction conceptuelle latente. Warburg l' a vait accentuée en s'appropriant l'usage de la grisaille ${ }^{21}$. Les Flo rentins plaçaient une grisaille antique en marge de leur prop re scénographie : en médaillon, en prédelle ou en pendentif. Comme les citations latines dans Montaigne, ces grisailles parlent différemment, sinon une autre langue, avec toutes les modalités possibles - d'exemplum, de médiation propitiatoire, d'ancestralité mythique ou de destin dont elles préfixent la scène principale.

En outre, par définition de son être reproductif, la photographie ne dit jamais un présent. Elle a intériorisé les modalités «baudelairiennes " des Tableaux parisiens, combinant un irréel du présent, un possible aléatoire, et le savoir de l'un et de l'autre. Ce dont jouaient aussi les sarcophages disposés dans le narthex de l'église, parfois reconvertis en baptistères, ou balisant le chemin qui conduit à la nef. En seuil ou en marge, les images et gestualités païennes déposées sur le marbre blanc, relevées au crayon ou à la plume, reprises en grisaille dans l'imagerie renaissante, avaient transpiré dans la scène chrétienne avec toutes les nuances qui leur permettent d'être tantôt modèle, tantôt fantôme, et tantôt commentaire. Mais toujours, ce souvenir-là évitait explicitement la touche d'actualité, comme les Sybilles antiques, gravées sur le pavage de l'église d'Orvieto en manière d'Introït, rejoignaient les prophètes de l'Ancien Testament pour annoncer la venue du Messie. Déposée sur l'écran, Darstellung et non Vorstellung, la photographie n'est pas un substitut de l'œuvre d'art qu'elle reproduit, et n'y prétend surtout pas. Warburg avait voulu que Gertrud Bing l'accompagnât dans son voyage italien pour acquérir une expérience directe de l'Italie et de ses palais, complément nécessaire au talent sans égal que sa collaboratrice apportait à la création de la bibliothèque de Hambourg. La photographie, comme la grisaille, avait ôté à l'image sa magie pour en radiographier le palimpseste. Elle obligeait l'œil à replacer chacune d'elle sur le diagramme de ses potentialités. 
L'Atlas saurait donc piéger l'image par l'image. Il la contraint à réfléchir sur ses propres pouvoirs en les divisant. Il mise sur une metanoia qui saurait rejoindre dans l'image le processus mental dont provient la figuration. Les stoïciens en avaient déjà exposé l'effet théorétique, c'est-à-dire d'apaisement et de suspension de l'action, et Warburg l'a vait mentionné dans sa conférence d'introduction. Mais ici l'usage d'écrans noirs et de montages discontinus écartait aussi bien le panégyrique du monde que la fonction initiatique de la Galerie de tableaux de Philostrate ${ }^{22}$. La récente guerre, l'impossibilité d'en constituer la visibilité, réclamaient plus et autre chose. L'Amérique avait ouvert ses propres temps modernes en vivant, presque simultanément, la guerre de Sécession, la conquête des derniers réduits indiens, et l'invention de la photographie - une photographie qui avait déchiré le voile de Tanit du paysage champêtre et des temps coloniaux. L'Atlas saisissait, cartographiait l'affect en cet instant où un geste rituel lui ouvrait l'issue de la visibilité et de la figuration, où l'héritage païen lui prêtait encore et toujours ses formes archétypales, c'est-à-dire culturellement disponibles et travaillées. Poursuivre cette histoire aussi près de l'actualité qu'il lui serait permis, Warburg en fit son dernier mot d'homme de science et d'action, et sa dernière fête.

L'attirance de Warburg pour la liesse populaire quand furent signés les a c co rdsdu Latran, fête païenne où il s'engloutit pendant plusieurs heures, a plus d'une fois étonné (Momigliano 1986). Dans les derniers mois de sa vie, il conçut quelques projets politiques, fondés sur un irénique partage des pouvoirs spirituels et politiques, peut-être sur le patron de l'imperator et du pontifex maximus. L'histoireprochaine de l'Eu rope allait y apporter un cruel démenti et révéler de non moins cruelles compromissions. Mais l' Atlas montre encore autre chose. Quand Warburg y ajouta, sur l'écran 78, la photographie vue davion d'une foule déferlant dans les avenues de Rome, comme sortant des vomitoires du Colisée, ou cette procession de l'Eucharistie (79), comme tout juste déro ulée de la colonne trajane, il identifiait - pour le meilleur et pour le pire - le Pathosformel de ces temps modernes qu'il voyait venir. Le pire est connu. Disons le meilleur. Dans le film que Malraux tira de L'Espoir, tout un village, serpent noir sur un flanc de montagne chauffé à blanc par le soleil, descend sur des brancards improvisés les corps d'aviateurs républicains abattus au combat, célébrant à l'Antique et d'un même geste leur gloire et leurs funérailles. Satyajit Ray a repris autrement la séquence, et d' a u tres que lui à n'en pas douter ${ }^{23}$. Comme le geste du David à la fronde, les photographies romaines ajoutaient un schème fort et ambigu à la grammaire de l'émotion.

22. "Une histoire de fantômes pour grandes personnes ", disait Warburg de son Atlas. Voir Philostrate, La Galerie de tableaux, avec le commentaire de Goethe (1968). Il y a, dans les Affinités électives, des effets de grisaille, des récits enchâssés, des boîtes optiques et des fresques, mais aussi une analyse de la magie des images. Ottilie est bien une de ces figures ménadiques qui paie de sa vie, à l'inverse de la sagesse qu'enseigne la grisaille, sa propre dissolution dans l'artefact d'une fresque préraphaélite.

23. Longue séquence sur laquelle s'achève le film que Malraux tira lui-même de son livre L'Espoir. Une scène comparable, également puissante, se voit dans La Déesse de Satyajit Ray (1960). 


\section{Pathosformel et limites de l'expressionnisme}

Fuir vers les choses réelles et le savoir... Quel savoir et quel réel trouvera-t-on dans ces formules pathiques où Warburg identifia l'apport de Dürer à la Renaissance allemande, avant la Guerre de trente ans? L'Atlas montre que là où l'émotion prend figure, quand ses effigies sont notre conscience, tout le réel est précisément lové dans ce travail de savoir. Warburg n'ignorait nullement qu'il s'en fallait de peu pour que la Pathosformel se perdît dans sa rupture expressionniste. Tracer la limite, donner à l'affect une formule « arrachée à la fruste anarchie de la souffrance, à l'absence en elle de loi » ${ }^{24}$, l'Atlas aura sa sanction dans sa capacité à saisir l'actualité sans risquer sa propre défaite. On a vu que le second écran alphabétique, fixant les points mystiques du corps zodiacal, avait écarté l'image d'une Passion, pourtant brièvement montrée à Kreutzlingen. Deux Passions figureront beaucoup plus loin (écran 74, Les Trois croix, gravures de Rembrandt), illustrant les transferts entre l'Europe du Nord et l'Italie centrale. Quant à l'expressionnisme qui avait suivi la guerre il exaltait avec une même outrance les formes extrêmes de l'Ich Drama et le retable d'Issenheim. Grünewald y avait écrit en lettres capitales le renoncement du Baptiste: Illum oportet crescere, Me autem minui. L'injonction n'avait aucune limite - balance abyssale sans point d'équilibre. Le Christ aux escarres qu'avait décrit Huysmans montrait la décomposition des chairs plutôt que la Résurrection ${ }^{25}$. Cette descente dans le nihilisme, qui prit, dans les années 20, l'ampleur d'un événement national, éclaire par contraste les choix de Warburg, polarités et paradigmes, de la nymphe botticcellienne au groupe antique du Laocoon. Warburg avait intégré le groupe rhodien dans l'histoire culturelle du geste pathique. Dés 1488, une copie de dimension réduite avait été exhumée. Lorsque le groupe actuellement exposé fut mis à jour, en 1506, il le fut "à l'apogée d'une décadence baroque" (Warburg 1999b; Settis 1999). En le plaçant à la limite extrême des formules pathiques renaissantes, Warburg suggérait que son succès échappait à ce que la virtuosité de l'exécution ou le miracle de l'exhumation pourraient expliquer. Dernière image du pathétique hellénistique et païen, il apportait une alternative aux figures crucifiées, rivalisant avec elle dans le parallèle de la douleur absolue. Poursuivant le même argument, les écrans de l'Atlas ont donné la préférence au geste d'abandon repris du Méléagre antique dans les représentations renaissantes de la Mise au tombeau.

Hébreu d'origine, hambourgeois de cour, florentin d'adoption, disait Warburg de lui-même. Plutôt qu'une note personnelle, qui n'apprendrait pas grand-chose, la formule doit se lire comme un blason. Elle situe la bibliothèque de Hamburg, à laquelle il s'était identifié, sur le socle d'une histoire des religions quaucune

24. Benjamin à Schoen, 30.7.1917.

25. Le retable d'Issenheim est propriété du musée de Colmar, ville redevenue française en 1918. Il avait été envoyé à Munich pour restauration. Son retour à Colmar fut une affaire diplomatique orageuse. Cf. François-René Martin (1998 : 29). L'attraction du Christ de Grünewald était telle, dans les premières décennies du siècle, qu'une reproduction du retable figurait dans toute chambre d'étudiant. Pas plus qu'Elias Canetti, Benjamin n'y avait failli jusqu'au moment où il lui substitua l'Angelus Novus de Klee. Cf. également, sur le Ich Drama, John Willett 1970. 
mémoire de l'image ne pouvait récuser. Comme s'il y avait dans toute peinture le souvenir d'une carte du ciel, un premier paganisme du haut et du bas, les symétries du corps, et tous les tropismes de la pesanteur et du soleil dont la perspective avait capturé la scène sans les réduire. Kant avait bien identifié les axes directeurs de cette cartographie du mouvement. Il citait, vraisemblablement à propos de L'École d'Athènes, une remarque de Mengs «si on les regarde longtemps, ils paraissent marcher ${ }^{26}$. Laissant ici l'analytique du goût, il s'en remettait à un peintre qui parle d'un autre peintre. Cette École d'Athènes est reproduite sur l'écran 53. Per astra ad sphaeram, disait Warburg, le geste opposé des deux philosophes, Platon et Aristote, indiquant «une considération (Betrachtung), comme on peut l'attendre du paganisme savant".

Autant que les demi-allusions aux crises phobiques de Warburg, dont il n'y a pas à douter, il faut dire la volonté d'acier (eiserne Wille, dit Forster) qui l'a guidé dans la recherche des conditions de la subjectivité consciente. "Die Statik des Ich-Gefuhls abhängig von der "Völkerwanderung" vorgeprägter Dynamogramme $»^{27}$. Le sujet d'expérience fait droit à une organisation psychique où l'émotion retrouve son étymologie de mouvement - fuite ou attirance. Peut-être Warburg a-t-il rendu quelque chose à notre cerveau nomade, archéologiquement sous-jacent au paganisme, articulant un réseau d'images et de cartes, virtuellement non clos. Kurt Forster note que la résurgence de ces schèmes de mouvement, dont Warburg poursuit la métamorphose et le déplacement, est bien l'énigme de la perception humaine ${ }^{28}$. Moins de dix ans plus tard, MerleauPonty substituera à ce patron perceptif qu'enseignaient les philosophes, ici encore néostoïcien, les médiations de la peinture. La tableau est le nœud où se nouent et se dénouent incessamment les cartes duvisible et du mouvenent, nouveau retable pour ce sentiment de l'existence qui fut, dans l'entre-deux-guerres et quelques décennies ensuite, le rituel du moderne ${ }^{29}$. Pour sa part, Warburg était persuadé que la physiologie du cerveau offrirait un jour les moyens scientifiques d'expliquer les effets les plus vifs et les plus détournés de l'émotion. Où il se pourrait que le contact entre le système neurosensible et l'environnement ne se diversifie en images qu'à faire jouer dabord sa protection émotionnelle, et à l'objectiver dans l'épaisseur des formes symboliques (Damasio 1995 ; LeDoux 1996; Vincent 1986). Warburg fait voir qu'il y avait une formule du pathos comme une formule de l'ethos, et que quelque part elles devaient coïncider si la culture est jamais possible. La Pathosformel aura donc relié, par le biais de nos corps rituels, la philosophie à l'anthropologie.

26. Kant $1970: \$ 13$. C’est à cette fresque, où Raphaël aurait exposé le programme d'un christianisme platonicien, que Wind consacra ses derniers travaux.

27. D'un des derniers carnets de Warburg, classé Allgemeine Ideen.

28. K. Forster 1999: 15. Ernst Gombrich a cité plusieurs notes du jeune Warburg qui tente d'écrire les moments successifs de la perception comme autant d'étapes aboutissant à une phénoménologie kantienne de l'expérience (Gombrich 1970 : 77-78). Ce à quoi le Pathosformel opposerait une réponse qui ouvrait la spécificité du champ de l'histoire de l'art. Ces hypothèses de départ seront clairement subverties par la diversité des documents iconographiques reproduits ensuite sur les panneaux de Mnemosyne. 29. Merleau-Ponty 1964 : chap. I et III. On reviendra sur ce texte testamentaire (1961) par référence auquel fut contrainte de se définir la philosophie française des trois décennies suivantes. 
Warburg fut avec Cassirer, au nombre de ceux qui prirent le criticisme sérieusement, en refusant la dérive du goût vers l'esthétisme. Mais, par un tour inverse à celui de Cassirer qui élaborait en concepts les articulation mythiques, Warburg déporte les expressions kantiennes sur leurs rebords interdits, comme on a vu pour le Pathosformel. Outrepassant cette séquence courte tracée par Heine, où Kant achèverait l'œuvre de Luther, Warburg donne la première place à Dürer, dans une histoire où Kant suit Dürer et Luther, mais enferme l'héritage plus qu'il ne l'accomplit (Heine 1998 [1855]). Tout à l'inverse, analysant Melancolie I et le " mysticisme transcendantal " de Luther, Warburg mit en cause cette aimantation de la Réforme vers son accomplissement de raison pure (Warburg 1990b : 277280). Dans les dernières pages de la Critique, Kant avait ébauché une Histoire de la raison, brève parce qu'aussitôt fermée, transformée en une architecture auquel le goût donne sa clé de voûte. En réponse, l'Atlas voulut servir une Kritik der reinen Unvernunft ${ }^{30}$. Ni véritablement histoire, ni véritablement philosophie, sa cartographie mentale se place au lieu même de la cartographie copernicienne du kantisme, organisée selon le même principe d'une analytique des fonctions. Ainsi Mnemosyne prendra un tout autre sens, une fois qu'on sera attentif à son dessein prospectif. L'Atlas sera d'autant plus clair qu'on le lira à rebours, du présent au passé, et des dernières images fixées sur la ligne basse des écrans vers les prototypes alexandrins, lesquels conferent, quand même et malgré tout, sa visibilité au présent. Cette anamnèse écarte autant l'impératif kantien, qui n'a jamais son présent d'accomplissement, qu'elle exclut l'infinitif expressionniste qui en serait la conséquence, toute de deuil et d'immobilité - Dasein. Ici, la survivance de l'Antique s'inverse. Le Moderne anime son présent par le mythe qu'il y accueille et qui le tire de son invisibilité sursaturée. "Autour de moi la ville, étourdissante, hurlait ", et c'est alors que la Passante baudelairienne organise l'innommable en "tableau parisien »: figure dansante comme celle casquée de fruits et de fleurs que Ghirlandaio introduit dans la naissance du Baptiste, y redistribuant la vie et les menaces de la vie. La figure indisciplinée prend sur elle ces éléments mythiques que Benjamin relevait dans Les Affinités électives. Goethe avait, lui aussi, introduit une dissonance dans le quatuor des amours disjointes, et un bougé dans la peinture. Lorsque Ottilie se prête au jeu du tableau vivant, où il lui fut dévolu de représenter la Madone Sixtine, l'enfant qu'elle porte sur son bras droit manque de lui échapper, comme il lui échappera quelques pages plus loin dans la tragique promenade en barque. Ce bougé est aussi l'impossibilité de (se) rejouer l'Antique que Raphaël avait pourtant voulu fixer dans un tableau qui donnait à la Vierge le déhanchement de l'Apollon du Belvédère. Mais se pouvaitil que cet emprunt ne déstabilise une scène si bien trichée d'avance? Warburg avait relevé, dans les mouvements superlatifs et inattendus de quelques personnages plus ironiques que véritablement ensauvagés, dans l'Intermezzo accompagnant les solennités princières, cela qui tout à la fois célèbre la fête et ne "tient " pas, et même dit en clair qu'elle ne tient pas. Le Moderne prendrait donc un

30. "Bilderatlas zur Kritik der reinen Unvernunft ", note tirée du cahier Grisaille, signalée par Charlotte Schoell-Glass (1991) 
risque nécessaire, que Warburg appelle paganisme, quand il joue de ses peurs et de quelques formules antiques pour inventer son présent.

Warburg découvrit avec enthousiasme que Manet, dans Le Déjeuner sur

l'herbe, avait composé un dessin de Raphaël représentant le Jugement de Paris, luimême inspiré d'un bas-relief et gravé par Raimondi, et le Concert champêtre de Giorgone. L'écran 55 réunit tous les éléments de ce transfert, en particulier le passage par le bas-relief et la gravure qui en redouble l'effet de grisaille. Manet avait non moins sûrement approprié quelque chose de l'effet de photographie et de citation à ses fins propres. Son tableau jouait d'une note d'irréalité et de scandale, préparant par d'autres moyens l'insolent discord que l'Olympia introduit dans le Pathosformel de l'odalisque. Dans les deux cas, il décapait le vernis antiquaire et faisait voir cette strate d'émotions dont était en quête la peinture française depuis Delacroix. Quant au Déjeuner, on reconnaittra, inséré sur la diagonale toute païenne qui traverse le tableau, une corbeille de fruits déposée sur l'herbe au bain lustral d'une moderne Nymphe dans le bosquet, en son centre, le double cadrage d'une quasi-photographie en noir et blanc et d'une scène d'atelier: ce trio posé, un nu (presque) académique et des figures masculines "hérö̈ques» dans leurs redingotes noires. Et toutes ces attitudes venues droit de l'Antique introduisent comme un soudain effet de "cliché" dans ce Déjeuner sur l'herbe - un trou d'anamnèse et d'irréel du présent dans l'économie de la toile. Warburg a bien relevé le cadrage savant dont il ne pouvait manquer de ressentir l'affinité avec ses propres montages. Il rejetait, non sans quelque jubilation d'initié, toute interprétation champêtre de la scène de plein air, inspirée de Rousseau ou des guinguettes. Quelle est, en effet, la temporalité de ce Déjeuner, sinon de faire voir dans cette archéologie du désir qu'il ne se passe rien, hormis une réplique ouvrant à un palimpseste, et que, tout de même, le reflet dans le miroir du Bar aux Folies Bergéres, répétait, avec le jeu d'irréalité des Ménines, son propre et éblouissant non-lieu ? La réminiscence cathartique vaudrait donc un irréel du présent, ponctuant la nouvelle relation du spectateur à l'image et l'évitement de la théâtralité interne au tableau qui ont été analysés par Michael Fried $^{31}$. Manet est bien en effet au point où se rencontrent le tableau et la muséographie qui institueraient, vers 1860 , la peinture dans son affect moderne.

Il est donc clair que l'Atlas ne convoque aucun primitivisme, ne propose aucune glorification du geste où l'hellénisme rejoindrait l'innocence d'une partie de campagne. "Le tragique du costume et de l'outil est l'histoire de la tragédie humaine, au sens le plus large, et le livre le plus profond qu'on ait écrit là-dessus est le Sartor Resortus de Carlyle » (cité in Gombrich 1970 : 221). Warburg s'intéressait aux vêtements et aux drapés antiques comme à la nécessité pour l'homme de gagner sa visibilité sur sa peur, son geste sur sa fuite, en cela comparable à la

31. Cf. La trilogie commencée avec La Place du spectateur (1990) et récemment conclue avec Le Modernisme de Manet (2000). Et cela par un historien de l'art philosophe qui étudia d'abord la peinture américaine, contemporaine, ainsi Morris Louis et Richard Serra, puis moderne, ainsi Thomas Eakins. Il avait accumulé une expérience permettant de comprendre comment la peinture française avait, entre David et Manet, transformé son rapport à l'Antique parce qu'elle avait rompu avec la scène de la phénoménalité et son corrélat d'imitation et de reconnaissance. 
nécessité biblique du travail. La peinture serait ce tégument de première vêture, où l'émotion s'arrache à l'inconscience, où le passage est accompli, plutôt que du toucher à la vue, du contact à l'intouchable du visible. Ne pas oublier que Delacroix, parti pour le Maroc avec l'ambassade du duc d'Aumale, croit voir la toge romaine dans le drapé des burnous, cherche le geste antique dans les échoppes marocaines, retrouve les Ménades dans les derviches tourneurs, note sur ses carnets les architectures et les couleurs, et peint à son retour La Noce juive et les Femmes d'Alger, ce tableau "qui nous guide assez vite vers les limbes insondés de la tristesse ». Baudelaire savait d'instinct que Delacroix avait donné ses formules d'émotion à la peinture d'après David. Mnemosyne, telle une Renaissance tirée de son événement daté, claire à elle-même et pour cela critique, à cent lieues d'une répétition "préraphaélite ", inventait une manière d'anamnèse mentale pour les temps que Warburg vivait ou voulait vivre. En incluant dans ses prototypes la Nymphe des Florentins, puis sa survivance dans la publicité des compagnies maritimes, sur les timbres-poste de la République française ou des lointaines provinces du Commonwealth, il soulignait qu'il n'était pas nécessaire d'en restreindre l'expression aux figures de l'extrême souffrance et du deuil. Il postulait qu'il devait être possible de renouer l'affect avec la vie civile, et que l'affect était le premier commanditaire de toute cette longue histoire.

L'histoire de la figuration, per monstra ad astra serait donc analogue à ce Sens unique où Walter Benjamin (1980) relevait au passage l'enseigne d'une cartomancienne: Madame Ariane deuxième cour à gauche, continuait son chemin et déchiffrait le dernier poteau indicateur:

"Vers le Planétarium:

Rien ne distingue davantage l'homme antique de l'homme moderne que son abandon à une expérience cosmique que ce dernier connaît à peine.

Et pourtant : elle s'impose de nouveau à chaque époque, et les peuples et les races lui échappent bien peu, comme on l'a vu lors de la dernière guerre, qui fut une tentative pour célébrer de nouvelles noces, encore inouïes, avec des puissances cosmiques. »

Ce planétarium, peut-être la dernière initiative civile et somptuaire de la République de Weimar, fut aménagé à Hambourg sur le site d'un château d'eau. En mars 1930, il accueillit l'exposition Sternglaube und Sternkunde, une conjonction entre deux termes qui ne se recouvriront jamais si l'histoire s'entretient de leur tension.

Warburg souhaitait que la confrontation de ses thèses avec l'anthropologie de l'Amérique précolombienne et moderne en éprouve la valeur scientifique. Quand il partit pour Rome, en 1929, il renonçait à un voyage aux États-Unis pour raison de santé. Un mémoire en a gardé le projet. On sait aujourd'hui que Warburg n'avait pas cessé, depuis 1895, de correspondre avec Franz Boas et les anthropologues américains. La question n'est pas ici d'apprécier ses chances d'être entendu Outre-Atlantique ni d'insérer de plein droit l'Atlas d'images dans les annales de l'anthropologie. Elle est de comprendre un propos moderniste qui mesure le présent de l'Allemagne aux diverses figures de la Renaissance et compare ce même propos à celui d'une anthropologie américaine qui eut à se définir entre le moder- 
nisme des grandes villes et l'attention aux "paganismes » indiens, enjambant de ce fait les limites philosophiques de l'héritage européen ${ }^{32}$. Boas reformulait une question ouverte au XVI ${ }^{\mathrm{e}}$ siècle, quand Montaigne avait reconnu combien peu la

Renaissance avait préparé les esprits aux réalités amérindiennes. On saurait au $\mathrm{XX}^{\mathrm{e}}$ siècle qu'elle affectait les deux termes de la confrontation.

Les écrans de l'Atlas furent-ils inspirés des collages de Lissitzky ou par les autels des Indiens pueblos? Répliquaient-ils les montages photographiques des affiches de Radtchenko ou une page de l'En cydopédie dadaïste ? Étaient-ils ro ulés et déroulés, comme les tapisseries flamandes avaient exporté leurs motifs du nord au sud de l'Europe ? Warburg achevait-il une carrière d'historien ou s'étaitil engagé dans l'ethnologie du Moderne? Kurt Forster n'a pas choisi, et il est essentiel de ne pas vouloir trancher. Le déploiement de l'image selon plusieurs axes analytiques et plusieurs stratégies figuratives fut une des multiples manières selon lesquelles la philosophie de l'art, dans les premières décennies du siècle, s'était frayé une issue.

Tout en soulignant le puissant affect investi dans ces cartes du ciel et coupoles de monde, au moment où l'Eu rope centrale était précisément en train de les perd re, l' Atlas transformait le rituel en une forme insolite de l'exposition, commémorant peut-être l'effort de ce géant d’avant les Olympiens que le mythe disait nécessaire afin que le ciel ne s'effondrepas sur les hommes. Il se pourrait aussi que, depuis ces années d' a vant-guer reoù on a tant disputé del'accrochage, nos musées aient restitué quelque chose de la valeur cultuelle en captant, mais par un détour institutionnalisé, une émotion deux fois modulée dans l'image et dans la demi-solennité des cimaises monochromes. Quasi indifférents à l'espace des salles, elles imposent leur parcours ou leur labyrinthe. Forme extrême et provocatrice de l'intrusion d'un espace dans un autre espace, l'installation déconcerte. Elle dissout son motif dans sa tension émotive et cryptée, laissant à qui s'en approche le soin d'entrer (ou non), plus que dans son informulé ou son informulable comme on dit, dans le laboratoire de son formulaire.

Moins de dix ans plus tard, Picasso, qui semblait ignorer tout de Warburg, jetait son bestiaire mythologique, noir sur blanc, à même l'écran de toile, sans haut ni bas, étiré comme un photogramme démesuré. Plus de ciel, ni sol ni sombra. Mais parce qu'il faut montrer et regarder quand même, deux lampes y font rentrer le jour. Elles éclairent des corps, désarticulés, hennissant, soufflés du centre vers la périphérie.

Et le public de l'Exposition universelle de 1937 apprit sur le champ ce qu'était Guernica.

MOTS CLÉSIKEYWORDS: Aby Warburg - anthropology de l'imagel anthropology of images - histoire de l'art/history of art - paganisme/paganism - renaissance - Pathosformel - modernitélmodernity.

32. Cf. "On Planned American Visit» (1927), in Michaud 1998 : Annexe II, 281-285. Voir également Forster 1991 : 11. Kurt Foster a relevé la coïncidence entre le programme de Boas (1982 [1902]) et les recherches historiques de Warburg. 
Barasch, Moshe

1991 "Pathos Formulae. Some Reflexions

on the Structure of a Concept ", in Moshe

Barasch, Imago Hominis. Studies in the

Language of Art. Vienne, IRSA.

\section{Bataille, Georges}

1952 Le Bleu du ciel. Paris, Gallimard.

\section{Benjamin, Walter}

1978 Correspondance, 1910-1940. Ed. et annoté par Gershom Scholem \& Theodor W. Adorno. Paris, Aubier Montaigne.

1988 Sens unique (1928) [précédé de] Enfance berlinoise [et suivi de] Paysages urbains. Trad. de l'allemand par Jean Lacoste. Paris, Maurice Nadeau.

1991 «Ursprung des deutschen

Trauerspiels ", in Gesammelte Schriften. Frankfurt am Main, Suhrkamp.

Boas, Franz

1982 "The History of Anthropology" [Address to the International Congress of Arts and Sciences, Saint Louis (1904)], in George W. Stocking, Jr, ed., A Franz Boas Reader: The Shaping of American Anthropology, 1883-1891. Chicago, University of Chicago Press : 23-36.

Burckhardt, Jacob

2001 Considérations sur l'histoire universelle. Paris, Éditions Allia.

\section{Damasio, Antonio R.}

1995 L'Erreur de Descartes. La raison des émotions. Paris, Odile Jacob.

Forster, Kurt W.

1991 «Die Hamburg-Amerika line, oder : Warburg's Kulturwissenschaft Zwischen den Kontinenten ", in Aby Warburg, Akten des Internationalen Symposium 1990. Weinheim, $\mathrm{VCH}$, Acta Humaniora.

1996 "Aby Warburg, his Study of Ritual and Art on Two Continents ", October 77 : 5-24. 1999 "Introduction », Aby Warburg, The Renewal of Pagan Antiquity. Los Angeles, Getty Research Institute («Texts \& Documents ») : 1-75.
Fried, Michael

1990 La Place du spectateur. Esthétique et origines de la peinture moderne. Paris, Gallimard.

2000 Le Modernisme de Manet, ou Le visage de la peinture dans les années 1860. Paris, Gallimard.

\section{Gombrich, Ernest H.}

1970 Aby Warburg, An Intellectual

Biography. London, The Warburg Institute.

Heine, Heinrich

1998 De l'Allemagne (1855). Paris,

Gallimard (« Tel»).

\section{Kafka, Franz}

1980 «Les Aéroplanes à Brescia» (1912), in Euvres complètes. Paris, Gallimard (« La Pléiade»), II : 110-120.

Kant, Immanuel

1952 Fondements de la métaphysique des mours. [Grundlegung zur Metaphysik der Stitten, 1785]. Trad. Victor Delbos. Paris, Delagrave.

1965 Critique de la faculté de juger. [Kririk der Urteilskraft, 1790]. Trad. Alexis Philonenko. Paris, J. Vrin («Bibliothèque des textes philosophiques").

1967 Prolégomènes à toute métaphysique future. [Prolegomena zu einer jeden künstigen Metaphysik die als Wissenschaft wird austreten Können, 1781]. Trad. par Jean Gibelin. Paris, J. Vrin ("Bibliothèque des textes philosophiques").

1970 Anthropologie du point de vue pragmatique. Trad. par Michel Foucault. Paris, J. Vrin ("Bibliothèque des textes philosophiques").

LeDoux, Joseph E.

1996 The Emotional Brain: The Mysterious Underpinnings of Emotional Life. New York, Simon \& Schuster. 
Lévi-Strauss, Claude

1962 Le Totémisme aujourd'hui. Paris, PUF

("Mythes et religions").

Martin, François-René

1998 "Images pathétiques ", Cahiers

du Cnam 63.

Merleau-Ponty, Maurice

1964 L'Eil et l'esprit. Paris, Gallimard.

Michaud, Philippe-Alain

1998 Aby Warburg et l'image en mouvement. Préface de Georges Didi-Huberman, suivi de Souvenirs d'un voyage en pays pueblo, 1923 [et] Projet de voyage en Amérique, 1927. Paris, Macula.

\section{Momigliano, Arnoldo}

1986 «How Roman Emperors Became Gods ", American Scholar 55.

\section{Philostrate l'Athénien}

1968 Die Bilder [von] Philostratos. Ed. par Otto Schönberger. Munich, E. Heimeran.

\section{Riegl, Alois}

1984 Le Culte moderne des monuments: son essence et sa genèse. Paris, Éditions du Seuil («Espacements").

\section{Schoell-Glass, Charlotte}

1991 «Warburg über grisaille : Ein Splitter über einen Splitter », in Horst Bredekamp, Michael Diers \& Charlotte Schoell-Glass, eds, Aby Warburg: Akten des Internationalen Symposium, Hamburg 1990. Weinheim, VCH, Acta Humaniora : 199-212.

\section{Settis, Salvatore}

1997 "Pathos und Ethos, Morphologie und Funktion ", in Wolfgang Kemp et al. eds, Vorträge aus dem Warburg-Haus. Berlin, Akademie Verlag, I : 31-73.

1999 Laocoonte, fama e stile. Roma, Donzelli («Saggi. Arti e lettere»).
Italo Spinelli \& Roberto Venuti, eds

1998 Mnemosyne. L'Atlante della memoria di Aby Warburg. Rome, Artemide.

Steinberg, Michael

1995 "Warburg's Kreutzlinger Lecture ", in Aby Warburg, Images from the Region of the Pueblo Indians of North America. Ithaca, Cornell University Press.

\section{Vincent, Jean-Didier}

1986 Biologie des passions. Paris, Éd. du Seuil.

Warburg, Aby

1938-1939 "A Lecture on Serpent Ritual », Journal of the Warburg Institute 2 : 222-292.

1988 Schlangenritual: ein Reisebericht.

Berlin, K. Wagenbach («Kleine kulturwissenschaftliche Bibliothek»).

1990a Essais florentins. Paris, Klincksieck.

$1990 \mathrm{~b}$ "La divination antique et païenne dans les écrits et les images à l'époque de Luther " (1920), in Essais florentins...

1990c «L'art du portrait et la bourgeoisie florentine » (1902), in Essais florentins... : 101-136.

1995 Images from the Region of the Pueblo Indians of North America, transl. with an interpretive essay by Michael P. Steinberg. Ithaca, Cornell University Press.

1998 Mnemosyne-Bilderatlas. Hambourg, Dölling und Galitz.

1999a «Pagan-Antique Prophecy in Words and Images in the Age of Luther ", in Aby Warburg, The Renewal of Pagan Antiquity. Los Angeles, Getty Research Institute («Texts \& Documents») : 597-698.

1999b "Dürer and Italian Antiquity " (1905), in Aby Warburg, The Renewal of Pagan Antiquity... : 553-558.

2000 Der Bilderatlas Mnemosyne, ed. par Martin Warnke avec la coll. de Claudia Brink. Berlin, Akademie Verlag («Studienausgabe»).

Willett, John

1970 L'Expressionisme dans les arts, 19001968. Paris, Hachette ("L'Univers des connaissances "). 
Claude Imbert, Warburg, de Kant à Boas. — Si l'histoire de l'art s'est renouvelée de par son alliance avec l'anthropologie, elle le doit en partie à la rencontre entre Warburg et Boas, deux transfuges de l'idéalisme allemand. Warburg a restitué le paganisme antique sous les canons alexandrins et renaissants. Son œuvre permet, par comparaison, d'approcher différemment cette modification qu'on a décrite, à partir de Baudelaire et Manet, comme modernité en peinture.
Claude Imbert, Warburg, from Kant to Boas. The history of art has been regenerated thanks to its alliance with anthropology, an alliance to be set down to the meeting between Warburg and Boas, who both deserted German idealism. Warburg restored ancient paganism using alexandrine and Renaissance canons. His work helps us, by way of comparison, to grasp in a different way this modification that, since Baudelaire and Manet, has been described as modernity in painting. 